\title{
Differential Emission Measures from the Regularized Inversion of Hinode and SDO data
}

\author{
I. G. Hannah and E. P. Kontar
}

SUPA School of Physics \& Astronomy, University of Glasgow, Glasgow, G12 8QQ, UK

Received ; Accepted

\begin{abstract}
Aims. To demonstrate the capabilities of regularized inversion to recover Differential Emission Measures (DEMs) from multiwavelength observations provided by telescopes such as Hinode and SDO.

Methods. We develop and apply an enhanced regularization algorithm, used in RHESSI X-ray spectral analysis, to constrain the ill-posed inverse problem that is determining the DEM from solar observations. We demonstrate this computationally fast technique applied to a range of DEM models simulating broadband imaging data from SDO/AIA and high resolution line spectra from Hinode/EIS, as well as actual active region observations with Hinode/EIS and XRT. As this regularization method naturally provides both vertical and horizontal (temperature resolution) error bars we are able to test the role of uncertainties in the data and response functions.

Results. The regularization method is able to successfully recover the DEM from simulated data of a variety of model DEMs (single Gaussian, multiple Gaussians and CHIANTI DEM models). It is able to do this, at best, to over four orders of magnitude in DEM space but typically over two orders of magnitude from peak emission. The combination of horizontal and vertical error bars and the regularized solution matrix allows us to easily determine the accuracy and robustness of the regularized DEM. We find that the typical range for the horizontal errors is $\Delta \log T \approx 0.1-0.5$ and this is dependent on the observed signal to noise, uncertainty in the response functions as well as the source model and temperature. With Hinode/EIS an uncertainty of $20 \%$ greatly broadens the regularized DEMs for both Gaussian and CHIANTI models although information about the underlying DEMs is still recoverable. When applied to real active region observations with Hinode/EIS and XRT the regularization method is able to recover a DEM similar to that found via a MCMC method but in considerably less computational time.

Conclusions. Regularized inversion quickly determines the DEM from solar observations and provides reliable error estimates (both horizontal and vertical) which allows the temperature spread of coronal plasma to be robustly quantified.
\end{abstract}

Key words. Sun:Corona - Sun:Flares - Sun: X-rays, gamma rays - Sun:activity - Sun:UV radiation

\section{Introduction}

Observations of the solar atmosphere with temperature sensitive spectral lines provide crucial information about the temperature distribution of the emitting plasma. These are vital for trying to resolve the question of which mechanisms heat different solar phenomena. Such as whether coronal loops are heated by a nanoflare model of magnetically reconnecting multibraided loop strands (e.g. Parker 1988) or chromospheric evaporation (e.g. Hirayama 1974). Or is the hot emission observed in large through to micro- flares (Fletcher et al. 2011; Hannah et al. 2011) predominantly due to energetic particles or other mechanisms such as waves. To reliably answer these questions, one needs to know not only the uncertainties on the emission for a given temperature, but also the uncertainties on the temperature itself, i.e. the temperature resolution.

The observations made with Hinode's X-ray Telescope XRT (Golub et al. 2007) and EUV Imaging Spectrometer EIS (Culhane et al. 2007) and SDO's Atmospheric Imaging Array AIA (Lemen et al. 2011) and EUV Variability Experiment EVE (Woods et al. 2010) provide a wealth of information about the solar emission over a broad range of temperatures. Assuming this UV/EUV/X-ray emission is both optically thin and in thermal equilibrium, via collisions, then the temperature distribu-

Send offprint requests to: Hannah e-mail:
iain.hannah@glasgow.ac.uk

tion of plasma emitting along the line of sight $h$ can be described by the differential emission measure DEM, typically given by $\xi(T)=n^{2} d h / d T\left[\mathrm{~cm}^{-5} \mathrm{~K}^{-1}\right]$ where $n(h(T))$ is the electron density at $h$ and with temperature $T$ (see Chapter 4 Mariska (1992) for detailed discussion of the different DEM forms). This however, can not be immediately inferred from such multiwavelength observations as the DEM is convolved by the emission processes and the instrumental response, i.e.

$g_{i}=\int_{T} K_{i}(T) \xi(T) d T+\delta g_{i}$

where $g_{i}$ is our observable for the $i^{\text {th }}$ filter, which has a temperature dependent response function $K_{i}(T)$ and $\delta g_{i}$ is the error. For spectroscopic observations this is respectively the line intensity and contribution function (examples shown in Figure 11. The uncertainties associated with these observations (counting statistics, background and instrumental errors) compounds the difficulty in the determination of the DEM and results in an ill-posed inverse problem (Tikhonov 1963; Bertero et al. 1985; Craig \& Brown 1986; Schmitt et al. 1996). Any direct attempts to solve Eq. 1. normally leads to the amplification of the uncertainties, and hence spurious solutions.

To reconstruct the DEM additional information (i.e. constraints) has to be added and numerous approaches have been developed to solve this problem (for overviews see, for instance, 
Monsignori Fossi \& Landini (1991) or Chapter 5 Phillips et al. (2008)). The simplest of these is to assume that all the emission is at a single temperature (isothermal) with $\xi(T) \propto \delta\left(T-T_{0}\right)$ where $\delta(x)$ is the Dirac delta function. The ratio of emission between two filters is then equal to the ratio of the response functions at the isothermal temperature (e.g. Weber et al. 2005; Reale et al.2009). Dividing the observable by the response function $g_{i} / K_{i}$ and plotting this as a function of temperature, the intersect point of the different curves (EM loci curves) will give the isothermal temperature and emission measure (e.g. Schmelz et al.2011). Although this is a simple and computationally fast method it does require the isothermal assumption and if the DEM is multi-thermal this method will produce erroneous results.

Another approach is to forward fit a chosen model, minimising the differences in observable space. This has been implemented for a discretised spline model DEM (Monsignori-Fossi \& Landini 1992; Brosius et al. 1996; Parenti et al. 2000) and more recently using the IDL mpfit routine from SDO/AIA and Hinode/XRT in M. Weber's xrt_dem_i terative2 . prd1. (Weber et al. 2004; Golub et al. 2004). This iterative forward fitting approach has also been developed with multiple Gaussian model DEMs using the IDL POWELL routine (Aschwanden \& Boerner 2011). To estimate the error in the DEM with these methods a Monte Carlo approach is adopted, producing multiple realisations within a given noise range. These approaches will find parameters for the model DEM but requires an assumed model and can be computationally slow when error estimates are required. The Metropolis MCMC approach has also been used on recovering the DEM for a large set of EUV spectral lines (Kashyap \& Drake 1998), part of PINTofALE spectral analysis package. This will give a robust measure of the parameter probability space but again can be computationally intensive, especially if a large number of lines and model parameters are considered. Bayesian formalism has also been recently used in a Bayesian Iterative Method (BIM), successfully reconstructing DEMs from both simulated and observed data (Goryaev et al. 2010). The iterative mpfit method was compared to a maximum likelihood and a genetic algorithm technique, finding similar results between the approaches (Siarkowski et al. 2008). Another genetic algorithm approach, which involves a preconditioning step where the optimum subset of spectral lines are selected, was found to be more effective (McIntosh et al. 2000). Currently a Singular Value Decomposition (SVD) inversion approach is also under development by Weber for SDO/AIA data.

Regularized inversion methods introduce an additional "smoothness" to constrain the amplification of the uncertainties, allowing a stable inversion to recover the DEM solution (e.g. Craig 1977; Craig \& Brown 1986). This was demonstrated to have promise for solar observations by Craig (1977) and subsequently tested on simulated data by Monsignori Fossi \& Landini (1991). Several forms of regularized inversion - truncated (or "zeroth-order") SVD, second-order regularization and maximum entropy regularization - have also been tested using simulated EUV spectral line emission (Judge et al. 1997). Although they determined that these approaches were superior to the simple ratio method they found several problems with the regularized inversion: the smoothness criterion used may not be physically "appropriate"; the solutions are highly sensitive to uncer-

\footnotetext{
${ }^{1}$ Available in SolarSoftWare with the Hinode/XRT software \$SSW/hinode/xrt/idl/util/xrt_dem_iterative2.pro
}
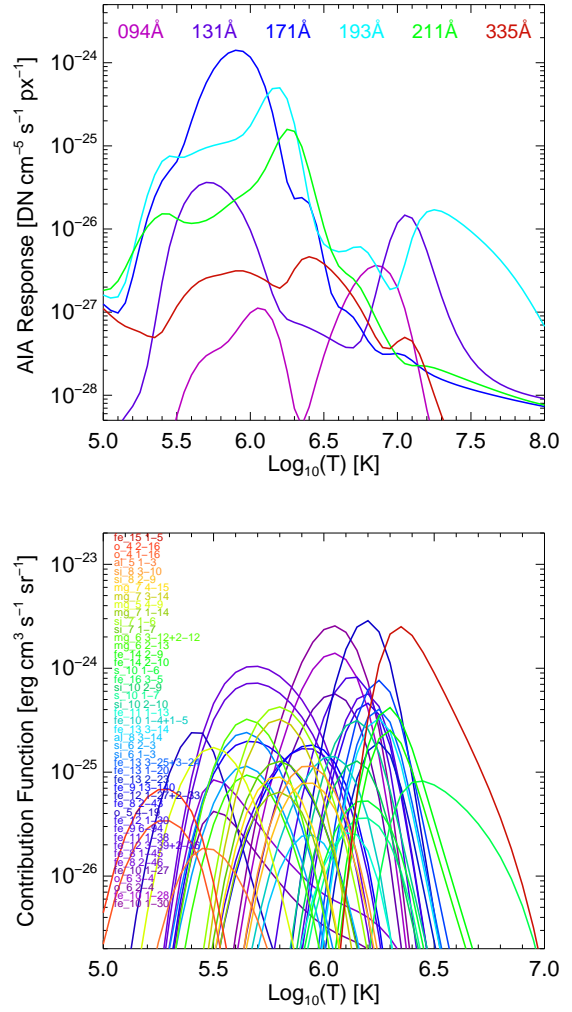

Fig. 1. Temperature response for SDO/AIA (Boerner et al.2011) and the contribution functions for several EUV lines from CHIANTI observable by Hinode/EIS (Landi \& Young 2009).

tainties in the kernel (K, response or contribution functions); the return of negative solutions.

In this paper we present a regularization method, which resolves some of these problems and robustly recovers the underlying DEM with errors. The method not only determines the DEM quickly, and its associated errors, but also naturally provides an estimate to the temperature resolution of the method. The ability to quickly compute (via Generalised Singular Value Decomposition) the DEM and its associated errors is due to the fact this method is linear, unlike those using maximum entropy for example. This method has already been implemented and applied to solar data for the inversion of RHESSI (Lin et al. 2002) X-ray spectra to their source electron distribution (Kontar et al.2004). Several other inversion techniques have been developed to infer X-ray photon spectra and/or electron spectra from RHESSI data (Piana et al. 2003; Massone et al. 2004; Brown et al. 2006) however it is the regularized inversion that has become the de facto approach. It has subsequently been used to infer the DEM, as well as the non-thermal emission, from RHESSI hard X-ray spectra (Prato et al. 2006).

In $\$ 2$ we detail the regularization method and how the errors and temperature resolution are determined. In $\S 3$ and $\S 4$ we demonstrate the capabilities of the regularization method, in comparison to other methods, on simulated SDO/AIA and Hinode/EIS data for a variety of model DEMs (single and multiple Gaussians and the CHIANTI DEM models). In $\$ 5$ we use the regularization method to recover the DEMs from observations of

2 The code written in IDL requiring $\mathrm{SSW}$ is available online: http://www.astro.gla.ac.uk/ iain/demreg/ 

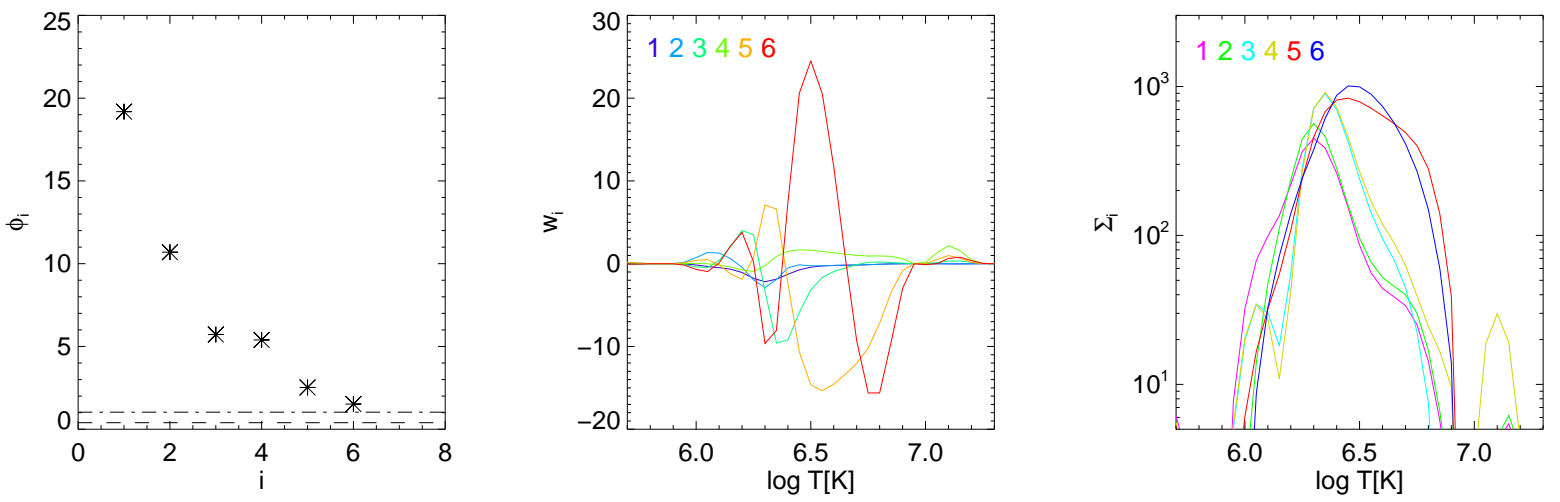

Fig. 2. Singular values $\phi_{i}$ (left), singular vectors $\mathbf{w}_{i}$ (middle) and the regularized solution Eq. 6 as a function of $i$ (right). The lines in the singular value plot (left) indicate the regularization parameter $\lambda$ that produces the solution with desired $\chi^{2}$ (dashed line) and with the additional positivity constraint (dash dot line). These values are from the regularized solution shown in Figure 3 .

an active region made with Hinode/EIS and XRT (Warren et al. 2010).

\section{Regularized inversion of multi-wavelength data}

To find the line of sight $\operatorname{DEM} \xi\left(T_{j}\right) j=1, \ldots, M\left[\mathrm{~cm}^{-5} \mathrm{~K}^{-1}\right]$ is to solve the system of linear equations:

$g_{i}=\mathbf{K}_{i, j} \xi\left(T_{j}\right)$

where $g_{i}$ is the observable (either the imaged DN or the integrated line intensity) for the specific filter or wavelength $i$ $(i=1, \ldots, N)$ and $\mathbf{K}_{i, j}$ is the corresponding temperature response or spectral line contribution function (examples shown in Figure 11. Eq. 2 is a generally ill-posed inverse problem and hence the least square problem

$$
\left\|\frac{\mathbf{K} \xi(T)-\mathbf{g}}{\delta \mathbf{g}}\right\|^{2}=\min ,
$$

does not have a unique solution. In the case $M=N$, a formal solution of Eq. 2 can always be written as $\xi\left(T_{j}\right)=\mathbf{K}_{i, j}^{-1} g_{i}$, where $\mathbf{K}^{-1}$ is the inverse of matrix $\mathbf{K}$. However, due to the large condition number, which is the ratio of the largest to the smallest singular values of $\mathbf{K}$, such a solution is meaningless in all practical cases due to substantial noise amplification (Craig 1977; Bertero et al. 1985). In addition, with $M>N$ the system is also under-determined. With inherent statistical and/or instrumental noise/uncertainties in the data $\mathbf{g}$, the information about the true solution $\xi(T)$ is lost and cannot be recovered without adding extra information about $\xi(T)$. Therefore constraints must be applied to obtain a unique meaningful solution. All methods solving this system to find the DEM $\xi(T)$ explicitly or implicitly add information not present in the data to obtain the approximate solution. The simplest, but most popular way to constraint the data is to fit a model function $\xi\left(T, \alpha_{i}\right)$ with a number of free parameters $\alpha_{i}$ that minimise the Eq. 3. Forward fitting is highly unsatisfactory if the functional shape of $\xi(T)$ is a priori unknown.

As any attempt to reconstruct the DEM directly leads to substantial noise amplification in $\xi(T)$, the broad approach to achieve a solution is to add linear constraints to the DEM (e.g. Tikhonov 1963; Bertero et al. 1985, 1988; Craig 1977; Craig \& Brown 1986). Often, so-called zero order regularization is used, which selects the smallest norm solution out of infinitely many possible solutions. This approach proved to be robust for various problems and is not over restricting (Bertero et al. 1985, 1988). Hence, we solve the least square problem

$$
\|\widetilde{\mathbf{K}} \xi(T)-\widetilde{\mathbf{g}}\|^{2}=\text { min subject to }\left\|\mathbf{L}\left(\xi(T)-\xi_{0}(T)\right)\right\|^{2} \leq \text { const, }
$$

with $\widetilde{\mathbf{K}}=(\delta \mathbf{g})^{-1} \mathbf{K}$ and $\widetilde{\mathbf{g}}=(\delta \mathbf{g})^{-1} \mathbf{g}$. This can be solved using Lagrangian multipliers, i.e.

$$
\|\widetilde{\mathbf{K}} \xi(T)-\widetilde{\mathbf{g}}\|^{2}+\lambda\left\|\mathbf{L}\left(\xi(T)-\xi_{0}(T)\right)\right\|^{2}=\min ,
$$

where $\mathbf{L}$ is the constraint matrix, $\lambda$ is the regularization parameter (related to the $\chi^{2}$ of the solution), and $\xi_{0}(T)$ is the "guess" solution, which will be explained in detail below. The L2 norm is defined as a sum $\|\mathbf{x}\|^{2}=\mathbf{x}^{T} \mathbf{x}=\sum_{i=1}^{N} x_{i}^{2}$ over all filters or intensities. Importantly, the solution of Eq. 5 is unique and wellbehaved. The formal solution of Eq. $5 \xi_{\lambda}(T)$ can be simply expressed in matrix form as a function of regularization parameter $\lambda$ but to avoid time consuming matrix manipulations Generalized Singular Value Decomposition GSVD (Hansen 1992) is used. The GSVD of matrices $\widetilde{\mathbf{K}} \in \mathbb{R}^{\mathbf{M} \times \mathbf{N}}, \mathbf{L} \in \mathbb{R}^{\mathbf{N} \times \mathbf{N}}$ produces a set of singular values $\gamma_{i}, \beta_{i}$ and singular vectors $\mathbf{u}_{i}, \mathbf{v}_{i}, \mathbf{w}_{i}$, with $i=1, \ldots, N$ which satisfy $\gamma_{i}^{2}+\beta_{i}^{2}=1, \mathbf{U}^{\mathbf{T}} \widetilde{\mathbf{K}} \mathbf{W}=\operatorname{diag}(\gamma)$ and $\mathbf{V}^{\mathbf{T}} \mathbf{L W}=\operatorname{diag}(\beta)$. These then provide the solution (Hansen 1992) to the minimization problem given in Eq. 5 as

$\xi_{\lambda}(T)=\sum_{i=1}^{M} \frac{\phi_{i}^{2}}{\phi_{i}^{2}+\lambda}\left(\frac{\left(\mathbf{g} \cdot \mathbf{u}_{i}\right) \mathbf{w}_{i}}{\gamma_{i}}+\frac{\lambda \xi_{0}(T)}{\gamma_{i}^{2}}\right)$,

with $\phi_{i}=\gamma_{i} / \beta_{i}$. This solution weights the contributions from various singular vectors differently, filtering out the singular vectors with $i$, for which $\phi_{i}^{2}<\lambda$. Hence removing un-physical oscillatory component of the solution (Bertero et al. 1988). Figure 2 shows typical behaviour of singular values and vectors as well as the construction of the solution.

\subsection{Regularization Parameter}

To find the solution $\xi_{\lambda}(T)$ is to determine the regularization parameter $\lambda$, which is done using Morozov's discrepancy principle (Morozov 1967), i.e.

$\frac{1}{N}\left\|\widetilde{\mathbf{K}} \xi_{\lambda}(T)-\widetilde{\mathbf{g}}\right\|^{2}=\alpha$, 
solving for $\lambda$ after substituting $\xi_{\lambda}(T)$ from Eq. 6. Here $\alpha$ is the regularization "tweak" value which effectively controls the required $\chi^{2}$ of the solution in observable space. The $\alpha$ value has a clear meaning when $\left\|\widetilde{\mathbf{K}} \xi_{\lambda}(T)-\widetilde{\mathbf{g}}\right\|^{2}$ are normally distributed, with a mean of $N$ and variance of $\sqrt{N}$. Therefore values of $\left\|\widetilde{\mathbf{K}} \xi_{\lambda}(T)-\widetilde{\mathbf{g}}\right\|^{2}$ in the range $N-\sqrt{N}<N+\sqrt{N}$ are acceptable values and $\alpha$ helps choose the exact value within this range. This also helps to put more or less weight on the data, with $\alpha<1$ requiring a "better" agreement with the data.

\subsubsection{Positively defined DEM}

Using the method discussed in the previous section, it is possible to select only positive solutions from the family of solutions $\xi_{\lambda}(T)$ by choosing an appropriate $\lambda$. The method based on Morozov's discrepancy principle chooses the parameter $\lambda$ that gives the DEM solution $\xi_{\lambda}(T)$ with the desired $\alpha$ in Eq. 77 The intrinsic DEM from the Sun should be positive but the regularization method provides no guarantee of a positive solution. Although this appears to be a problem with this implementation of the regularization method, one should remember that the DEM derived from observations need not be positive given the often poorly known uncertainties, response functions and the possibility of background subtraction.

A positive DEM solution can be achieved with an additional criterion to the choice of regularization parameter. That is we take the regularization parameter $\lambda$ that provides the smallest $\left\|\widetilde{\mathbf{K}} \xi_{\lambda}(T)-\widetilde{\mathbf{g}}\right\|^{2}-\alpha N$ and $\xi_{\lambda}(T)>0$. This approach has the advantage of maintaining the linear calculation of the solution unlike those that try to implement the positivity constraint in Eq. 6 directly, producing non-linear or iterative solutions (e.g Piana \& Bertero 1997; de Villiers et al. 1999). As $\alpha$ is the $\chi^{2}$ of the regularized solution, the ability to recover a positive solution strongly depends on the error estimates on the input data and knowledge of $\mathbf{K}$. If the error used is too small the $\chi^{2}$ of a positive solution can be erroneously high and in general, the positivity constraint produces a larger $\chi^{2}$, behaviour previously demonstrated by Bertero \& Dovi (1981).

\subsection{Initial Guess Solution}

The standard mode of operation of our regularization algorithm requires no initial guess solution, i.e. $\xi_{0}(T)=0$. However, $\xi_{0}(T)$ is used in the calculation of the constraint matrix (either for the higher order constraints or in the constraint weighting, see $\$ 2.3$. To avoid this problem we run our regularization algorithm (solving Eq. 7 and then Eq. 6) twice. On the first run the guess solution is $\xi_{0}(T)=0$, the constraint matrix is the identity matrix $\mathbf{L}=\mathbf{I}$ and we find a weakly regularized solution with $\alpha=10$. The regularized solution found can then be used as the initial guess for the second run $\left(\xi_{0}(T)=\xi_{R}(T)\right)$ and in calculating the chosen constraint matrix (as discussed in \$2.3). For this second run a stronger regularization is used $\alpha=1$ to find the final solution, then the associated errors in the DEM and temperatures are calculated, see $\$ 2.4$.

An alternative approach can be taken when working with high resolution spectroscopic line data as we can use the minimum of the EM loci curves as the initial guess solution. The EM loci curve for each spectral line is the ratio of the line intensity to contribution function $\approx g_{i} / K_{i}$. As this estimates the EM based on the isothermal temperature, it provides the upper limit to the DEM as a function of temperature. Multiple spectral lines across a wide range of temperatures provides a strong constraint to the DEM space and a useful initial guess solution. When selected it is automatically calculated within our code and as we are starting with a non-zero guess solution, this approach only requires one run of the algorithm. These two approaches to the guess solution will be demonstrated for Hinode/EIS data, simulated from a variety of model DEMs, in $\$ 3.2$ and $\$ 4.2$. For broadband data (such as those from SDO/AIA), only the $\xi_{0}(T)=0$ approach is used as the minimum of the EM loci curves provides a poor guess solution, especially when the DEM is not isothermal.

\subsection{Constraint matrix}

There is a number of different choices for $\mathbf{L}$ and here we consider only linear constraints. Physically, the quantity $\int n_{e}^{2} d h=$ $\int \xi(T) d T$ is the total number of electrons along the line of sight, so $\|\xi(T)\|^{2} \leq$ constant, similar to the X-ray case (Piana et al. 2003). This corresponds to the constraint matrix of $\mathbf{L}_{0} \propto \mathbf{I}$, a zeroth-order constraint. So, applying zero order regularization, we find DEM with the smallest amount of plasma required to explain the observational data. The source averaged $\xi(T)$ results from a combination of heating, cooling and and the physics of heat transport in the radiating source. Therefore, when $\xi(T)$ is the solution of some differential equation, one can expect that $\xi(T)$ should be differentiable or equivalently the constraint matrix $\mathbf{L}$ is the first order or second order derivative $\left(\mathbf{L}_{1} \sim \mathbf{D}^{1}\right.$ or $\mathbf{L}_{2} \sim \mathbf{D}^{2}$ ), again similar to the X-ray case (Kontar et al. 2004). Hence higher order regularization constraints select solutions with the smallest variations in temperature and are more restrictive then zeroth-order solutions. The more restrictive methods put more weight on the a priori constraints rather than the analysed data sets, which could be advantageous for poorly measured data.

\subsection{Error and Temperature Resolution of DEM}

Suppose the true DEM solution $\xi_{\text {true }}(T)$ is given. Then we can write

$\mathbf{g}=\mathbf{K} \xi_{\text {true }}(T)+\delta \mathbf{g}$.

Any regularized solution to a linear problem can be viewed as the replacement of the generalized inverse $\mathbf{K}^{+}$with the regularized inverse $\mathbf{R}_{\lambda}$ (with $\lim _{\lambda \rightarrow 0} \mathbf{R}_{\lambda}=\mathbf{K}^{+}$), so that our regularized solution is $\xi_{R}(T)=\mathbf{R}_{\lambda} \mathbf{g}$. Indeed for $\xi_{0}(T)=0$, Eq. 8 can straightforwardly re-written in such form with $\mathbf{R}_{\lambda}$ expressed via known GSVD vectors and values and exists for all linear methods (Bertero et al. 1988). On the other hand, the true solution to Eq. 8 can be formally written $\mathbf{K}^{+} \mathbf{g}$. Therefore, to estimate the error we find the difference between the true solution and our solution

$\delta \xi(T)=\xi_{R}(T)-\xi_{\text {true }}(T)=\left(\mathbf{R}_{\lambda} \mathbf{K}-\mathbf{I}\right) \xi_{\text {true }}(T)+\mathbf{R}_{\lambda} \delta \mathbf{g}$

Eq. 9 shows the important result that the error comes from two parts: the last term gives us that the noise propagation (vertical errors) and the first term gives the temperature resolution (horizontal errors). This equation presents the method independent definition of both the horizontal and vertical resolutions. While the noise propagation is normally accounted by the DEM methods, the resulting temperature resolution is often not considered.

The error on the DEM $\delta \xi(T)$ (the vertical error $\mathbf{R}_{\lambda} \delta \mathbf{g}$ ) is calculated using the standard Monte Carlo approach (Press et al. 1992; Piana et al. 2003; Kontar et al. 2004; Prato et al. 2006) 

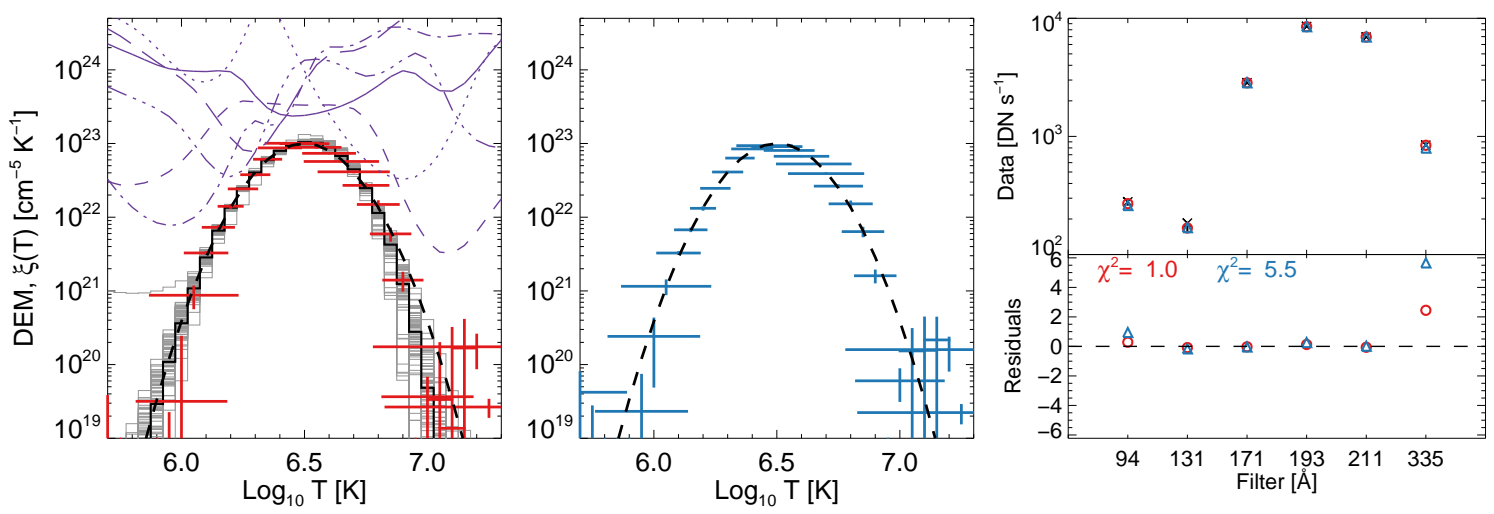

Fig. 3. Results of DEM reconstructions from simulated SDO/AIA data of a single Gaussian model. Shown is the zeroth-order regularized solution (left panel, red error bars) and with an additional positivity constraint (middle panel, blue error bars). The resulting simulated SDO/AIA data for each filter and the residuals of the regularized solutions are shown in the upper and lower right panels. Also plotted is the solution from xrt_dem_iterative2.pro (grey histogram, left panel) and the EM loci curves (purple lines, left panel).

with multiple random realisations of $\mathbf{g}$ within the noise range $\delta$. Then the one sigma spread of the regularized solutions from these realisations provides the measure of the uncertainty on the DEM. This is possible due to the linear nature of Eq. 9. In general, the exact statistics of the errors is needed but as this distribution is unknown, we assume a Gaussian distribution of standard deviation $\delta \mathbf{g}$ for each filter. Then the the vertical uncertainty on the DEM are calculated as the standard deviation using 300 MC Gaussian noise realisations. However, as the probability distribution of $\delta \mathbf{g}$ does not need to be Gaussian, the resulting DEM uncertainties could also be non-Gaussian.

The temperature resolution (horizontal error) of any linear inversion method - or temperature bias of the solution - is how much the product $\mathbf{R}_{\lambda} \mathbf{K}$ differs from the identity matrix $\mathbf{I}$. When the regularized inverse is similar to inverse of the kernel matrix $\mathbf{R}_{\lambda} \simeq \mathbf{K}^{+}$, the temperature resolution does not degrade giving $\mathbf{R}_{\lambda} \mathbf{K} \simeq \mathbf{I}$. However, for the ill-conditioned problem of DEM determination, $\mathbf{R}_{\lambda} \mathbf{K}$ is not identity matrix, but has a finite spread. The temperature resolution is then simply the FWHM of $\mathbf{R}_{\lambda} \mathbf{K}$ for a given temperature bin. This represents the temperature bias measure or the smallest temperature difference which can be meaningfully distinguished in the solution. Conveniently, $\mathbf{R}_{\lambda} \mathbf{K}$ is easy to calculate from the singular values and vectors obtained in GSVD decomposition of $\widetilde{\mathbf{K}}$ and $\mathbf{L}$ used to find the regularized solution. Namely, $\mathbf{R}_{\lambda} \mathbf{K}=\mathbf{W} \mathbf{Y} \mathbf{W}^{-1}$ where the column vector $\mathbf{w}_{i}$ forms matrix $\mathbf{W}$ and $\mathbf{Y}$ is a diagonal matrix with the elements constructed with singular values $\mathbf{Y}_{i i}=\gamma_{i}^{2} /\left(\gamma_{i}^{2}+\lambda \beta_{i}^{2}\right)$ (Kontar et al. 2004).

When $\mathbf{R}_{\lambda} \mathbf{K}=\mathbf{I}$ the response is diagonal or impulse-like for a given temperature, and therefore the true DEM $\xi_{\text {true }}(T)$ is not distorted. In any practical situation, $\mathbf{R}_{\lambda} \mathbf{K}$ is not diagonal but has off-diagonal elements (an example is shown in Figure 4 of a simulated DEM discussed in detail in \$3.1). The off-diagonal terms are a spread about a peak value at the diagonal (the red example in Figure 4), so for each temperature $T_{j}$ the row of $\left(\mathbf{R}_{\lambda} \mathbf{K}\right)_{i}$ has a finite width. The FWHM of this spread is then taken as the temperature resolution for that particular temperature. When the off-diagonal terms dominate (the blue and green examples in Figure 4), the FWHM can still be used to indicate the poor temperature resolution. It should be noted that $\mathbf{R}_{\lambda} \mathbf{K}$ depends on the errors $\delta \mathbf{g}$, so larger errors results in a poorer temperature resolution, e.g. wider row of the matrix $\mathbf{R}_{\lambda} \mathbf{K}$. The dependency on
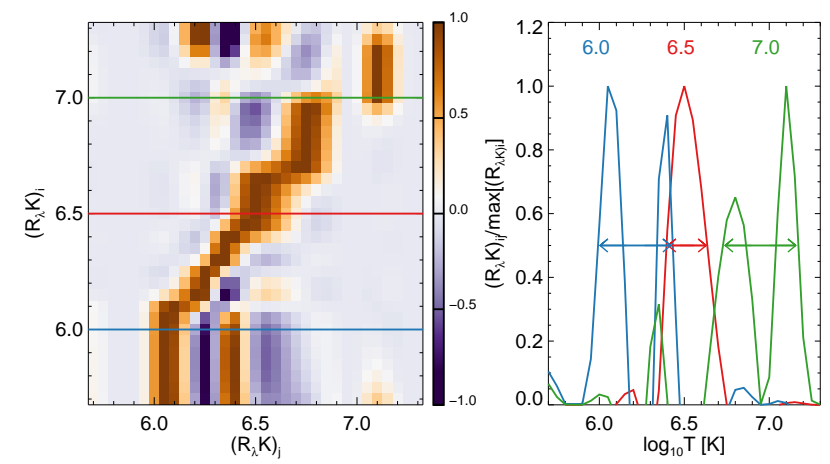

Fig. 4. (Left) $\mathbf{R}_{\lambda} \mathbf{K}$ matrix with each row providing the temperature resolution information for each temperature $T_{j}$. The highlighted rows are plotted in the right panel also showing the FWHM estimate used to calculate the temperature resolution.

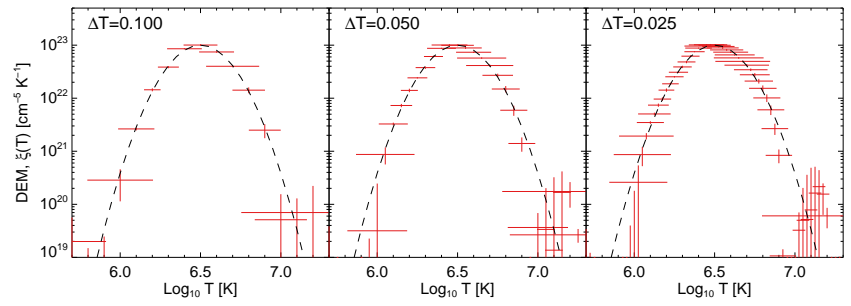

Fig. 5. Effect of changing the temperature bin size for the single Gaussian model DEM, shown in Figure 3.

$\delta \mathbf{g}$ comes via the regularization parameter $\lambda$ given by Eq. 7 . This definition of temperature resolution is more conservative as it does not assume a form of DEM as in Weber (2009).

\subsection{Temperature response and instrument uncertainties}

One of complicating factors is that the kernel (the matrix $\mathbf{K}$ ) of the integral equation to be inverted is only known to a limited degree. The errors come from the calibration uncertainty of the instrument itself and the uncertainty in the dominant spectral contribution of each bandpass (e.g. Aschwanden \& Boerner 2011; 
O'Dwyer et al. 2011). Therefore, the temperature response has an uncertainty $\delta \mathbf{K}$ and the linear problem, Eq. 8, becomes

$\mathbf{g}=(\mathbf{K}+\delta \mathbf{K}) \xi_{\text {true }}(T)+\delta \mathbf{g}$.

This translates into an additional uncertainty for $\xi(T)$ compared to Eq. 9

$$
\delta \xi(T)=\left(\mathbf{R}_{\lambda} \mathbf{K}-\mathbf{I}\right) \xi_{\text {true }}(T)+\mathbf{R}_{\lambda} \mathbf{K} \delta \mathbf{g}+\mathbf{R}_{\lambda} \delta \mathbf{K} \xi_{\text {true }}(T)
$$

When the uncertainty $\delta \mathbf{K}$ is dominated by a systematic error in the intensity of the lines measured then it is not temperature dependent but a constant scaling factor per filer or line (i.e. rows of K). Since the shape of the response or contribution function does not change as a function of temperature then this is identical to the introduction of an additional error to $\delta \mathbf{g}$ with Eq.11 reducing to Eq. 9. This will be investigated further in $\$ 3.2$ and $\$ 4.2$ with reference to Hinode/EIS.

\section{Simulated Data: Gaussian Model}

We test the regularization method on simulated SDO/AIA $(\$ 3.1$ and Hinode/EIS data (\$3.2) of Gaussian model DEMs. These have the form

$\xi\left(T_{j}\right)=\frac{N_{0}}{\sqrt{2 \pi} \sigma_{T}} \exp \left[\frac{-\left(\log T_{j}-\log T_{0}\right)^{2}}{2 \sigma_{T}^{2}}\right]$

where $\log T_{0}$ is the centroid temperature, $\sigma_{T}$ is the standard deviation and $N_{0}=\int \xi(T) d T$. We consider model DEMs of one to three Gaussian components. Note that as we calculate everything using $\log T$ instead of $T$, a conversion factor of $T \ln (10) d \log T$ is required in Eqs. 1 and 2 .

\subsection{SDO/AIA Simulated Data}

To simulate SDO/AIA data we take the model DEM from Eq. 12 and calculate the expected observable signal in each of AIA's six coronal filters $g_{i}$ using the response functions $K_{i}$ (Boerner et al. 2011), shown in the top panel of Figure 1 From this we calculate the associated error $\delta g_{i}$ using the readout noise and photon counting statistics (correcting from $\mathrm{DN} \mathrm{s}^{-1} \mathrm{px}^{-1}$ to photons via the electron and photon gains). Then Gaussian noise within these $\delta g_{i}$ is added to $g_{i}$. Our simulated observables $\mathbf{g}, \delta \mathbf{g}$ and the response functions $\mathbf{K}$ are the inputs to the regularization algorithm. As described in $\$ 2$ we run the regularization twice: on the first run we use $\alpha=10, \mathbf{L}=\mathbf{I}, \xi_{0}(T)=0$ which provides a guess solution that can be used to calculate the desired constraint matrix $\mathbf{L}_{\mathbf{0}}, \mathbf{L}_{\mathbf{1}}, \mathbf{L}_{\mathbf{2}}$ and final regularized solution with $\alpha=1$.

We first consider a single Gaussian model of $N_{0}=3.76 \times 10^{22}$ $\mathrm{cm}^{-5}, \sigma_{T}=0.15$ and $\log T_{0}=6.5$, shown in Figure 3. Here the regularized DEM (red error bars) was found using $\mathbf{L}_{\mathbf{0}}$ (zerothorder constraint) and well matches the original model DEM (black dashed line). In observable space (right panel in Figure 3) the residuals between the model and regularized solution are small with $\chi^{2} \approx 1.0$ close to the desired value set by $\alpha=1$. Here $\chi^{2}$ is taken as the sum of the square of the residuals divided by $N$ to match the version in Eq.7 The $\mathbf{R}_{\lambda} \mathbf{K}$ matrix for this regularization (Figure 4) is diagonal over approximately $\log T=6.1-6.9$. The horizontal error bars (temperature resolution) at each temperature bin is taken as the standard deviation of the FWHM of the rows of $\mathbf{R}_{\lambda} \mathbf{K}$. Outside this range the matrix is clearly not diagonal, producing large horizontal errors, so the regularized DEM is not reliable at these temperatures.
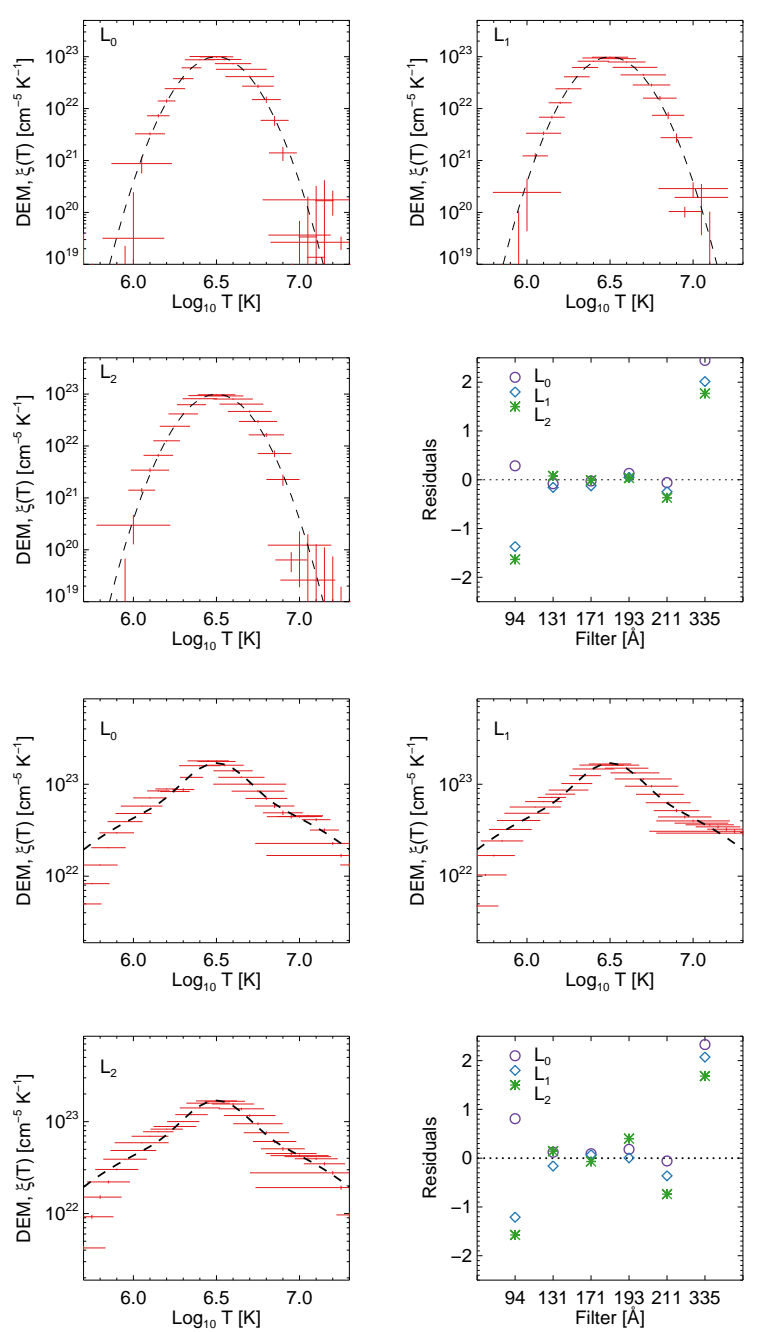

Fig. 7. Testing the effect of changing the constraint matrix or$\operatorname{der}\left(\mathbf{L}_{\mathbf{0}}, \mathbf{L}_{\mathbf{1}}, \mathbf{L}_{\mathbf{2}}\right.$, zeroth, first and second order respectively). The top four panels show the different recovered DEM and resulting residuals for the single Gaussian model shown in Figure 3 . The bottom four panels shows the same for the broad double Gaussian model shown in Figure 6

At the lowest temperatures $(\log T \leq 6.0)$ some negative DEM values are found, not shown on the log-scale, but this is in a temperature range where the DEM contribution is minuscule (over 4 orders of magnitude smaller than the peak) and the temperature response is very weak. At the highest temperature $(\log T \geq 7.0)$ we have positive regularized DEM but with large horizontal and vertical errors again due to being in a range where the DEM component is very small. In the middle panel of Figure 3 we show the regularized solution (blue error bars) in which the regularization parameter has been chosen to minimise $\chi^{2}$ to the desired value whilst forcing a positive DEM. The result is a very slightly different DEM, particularly below $\log T \approx 6.1$, and is well within the error bounds shown for both regularized solutions. As expected the $\chi^{2}$ of the solution is higher (5.5 instead of the desired 1) but within the DEM error bounds and so both are valid solutions.

For comparison we have calculated the DEM solution using the iterative forward fitting routine xrt_dem_iterative2 .pro (black histogram in left panel Figure 3) with 75 Monte Carlo (MC) realisations of the solution within the observable error 

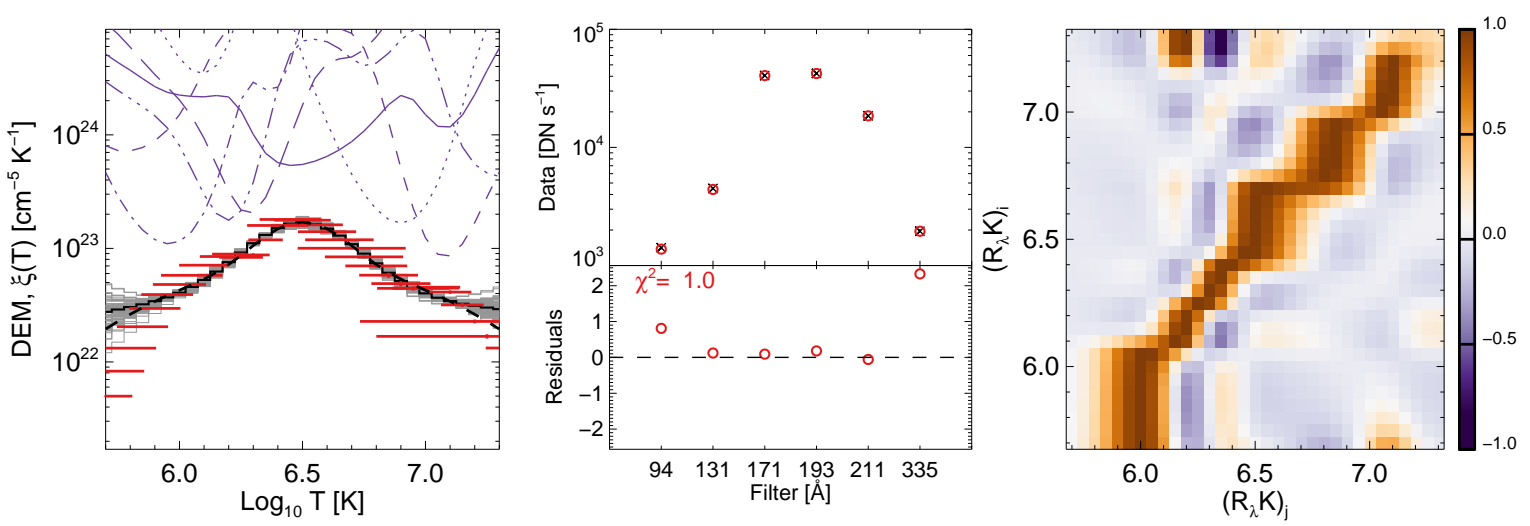

Fig. 6. Example DEM from zeroth-order regularization of simulated SDO/AIA from a two Gaussian model. (Left) DEM space, (middle) observable space and residuals (right) $\mathbf{R}_{\lambda} \mathbf{K}$ used to calculate the temperature resolution. Also plotted is the solution from xrt_dem_i terative2 . pro (grey histogram, left panel) and the EM loci curves (purple lines, left panel). Note that the solution here is positive without the additional constraint.

bound $g_{i} \pm \delta g_{i}$ (grey histograms). This also agrees well with the model DEM and also shows a larger spread in DEM solution in the temperature ranges where the regularized solution has large errors. Again this is due to the minor contribution to the DEM (about four orders of magnitude smaller than the peak value) in these temperature ranges. Also for comparison we have plotted the EM loci curves $\left(g_{i} /\left(K_{i} T d \ln T\right)\right)$ which do not intersect at a single point as this is not an isothermal model DEM. The DEM solutions are below the EM loci curves, as expected, since these estimate the upper limit of the emission.

The error bars shown for the regularized solution are not independent of each other and map out an error boundary region. This can be seen in Figure 5 where we show the regularized DEM using a variety of temperature binsizes. To achieve this we need to interpolate the temperature response functions (top panel Figure 1) from the original binning to our chosen temperature binsize. Increasing the number of temperature bins does not change the shape of the regularized DEM but produces a clearer definition of the error bound, with the overlapping error bars indicating that the nearby DEM bins are clearly not independent. This does however slow down the computation of the regularization process, although at worst it still only took a few seconds to compute. Note again that the vertical errors are taken as the variance of 300 regularized solutions found from random realisations within $g_{i} \pm \delta g_{i}$ and so we are explicitly assuming a simple Gaussian spread of vertical error in the regularized solutions. In reality the distribution of these errors will be more complicated.

A second example is shown in Figure 6 where an additional broad Gaussian component $\left(N_{0}=8.77 \times 10^{22} \mathrm{~cm}^{-5}, \sigma_{T}=0.5\right.$ and $\log T_{0}=6.5$ ) has been added to the model DEM shown in Figure 3. Immediately it is clear that the regularization produces a $\mathbf{R}_{\lambda} \mathbf{K}$ that is diagonal over a wider temperature range, indicating that the narrow temperature range found for the single Gaussian model (Figure 3) was mostly due to the DEM model dominating over a small temperature range. For the broader DEM model there are still deviations from a diagonal $\mathbf{R}_{\lambda} \mathbf{K}$ at the ends of the temperature range chosen but this is due to the limited response of AIA at these temperatures (see Figure 1). This time only the solution without a positivity constraint is shown since the regularization parameter for $\chi^{2}=1$ provides a positive solution. For comparison the forward fitted solutions from xrt_dem_iterative2.pro (grey histograms) are shown and again match the regularized solution (and model DEM) over the majority of the temperature range but show a wider verti- cal spread at the smallest and largest temperatures. This consistent increase in error in the DEM solution from both methods suggests the poor response at these temperature extremes is the source of this uncertainty. The EM loci curves are also shown but again do not intersect at a single point as this is a broad DEM.

In both examples shown so far only the zeroth-order constraint has been used and in Figure 7 we show the resulting regularized DEM for these cases for higher order constraint matrices. For the single Gaussian the higher order constraint removes the cluster of "noisy" data points at high temperature but also broadens the temperature resolution at around $\log T_{0}=6.2-6.4$. This shows that the constraint is changing the balance between these two temperature ranges and in this case the lower order constraint is preferable as it produces a better temperature resolution where the DEM is dominant. For the two Gaussian model DEM the higher order constraints again produce a broader temperature resolution which helps the regularized solution match the model better at low temperatures. Therefore in this case the higher order constraint is marginally preferable. As expected, in both cases the increase in order of the constraint matrix increases the "smoothing" of the regularized solution. In the subsequent analysis present here the zeroth order $L_{0}$ constraint matrix is used throughout as it is generally sufficient to recover the expected DEM.

We now consider the ability of the regularized inversion to recover single Gaussian model DEMs but with different widths and magnitudes, shown in Figure 8 . Here we do not use the positivity constraint since for narrow DEMs the possible negative regions are from temperatures where the contribution is tiny and consistent (within the errors) with zero and for broad DEMs the are no negative values recovered. As we set $\alpha=1$ the $\chi^{2}$ of the regularized solution is also approximately 1 . Firstly, we increase the width of the Gaussian from the minimum expected of $\sigma_{T}=0.1$, the designed achievable temperature resolution of SDO/AIA (Judge 2010), to $\sigma_{T}=0.4$, using a normalisation magnitude of $N_{0} /\left(\sqrt{2 \pi} \sigma_{T}\right)=10^{23} \mathrm{~cm}^{-5} \mathrm{~K}^{-1}$ in Eq. 12. shown in the top panels of Figure 8 The regularized inversion method is able to recover the model DEM at the limit of SDO/AIA's temperature resolution. For the widest model DEMs there is some slight deviation but it is consistent within the error bars. Again we compare the regularization method to xrt_dem_iterative2.pro (blue histograms) and find similar solutions, with both methods deviating from the model DEM in 

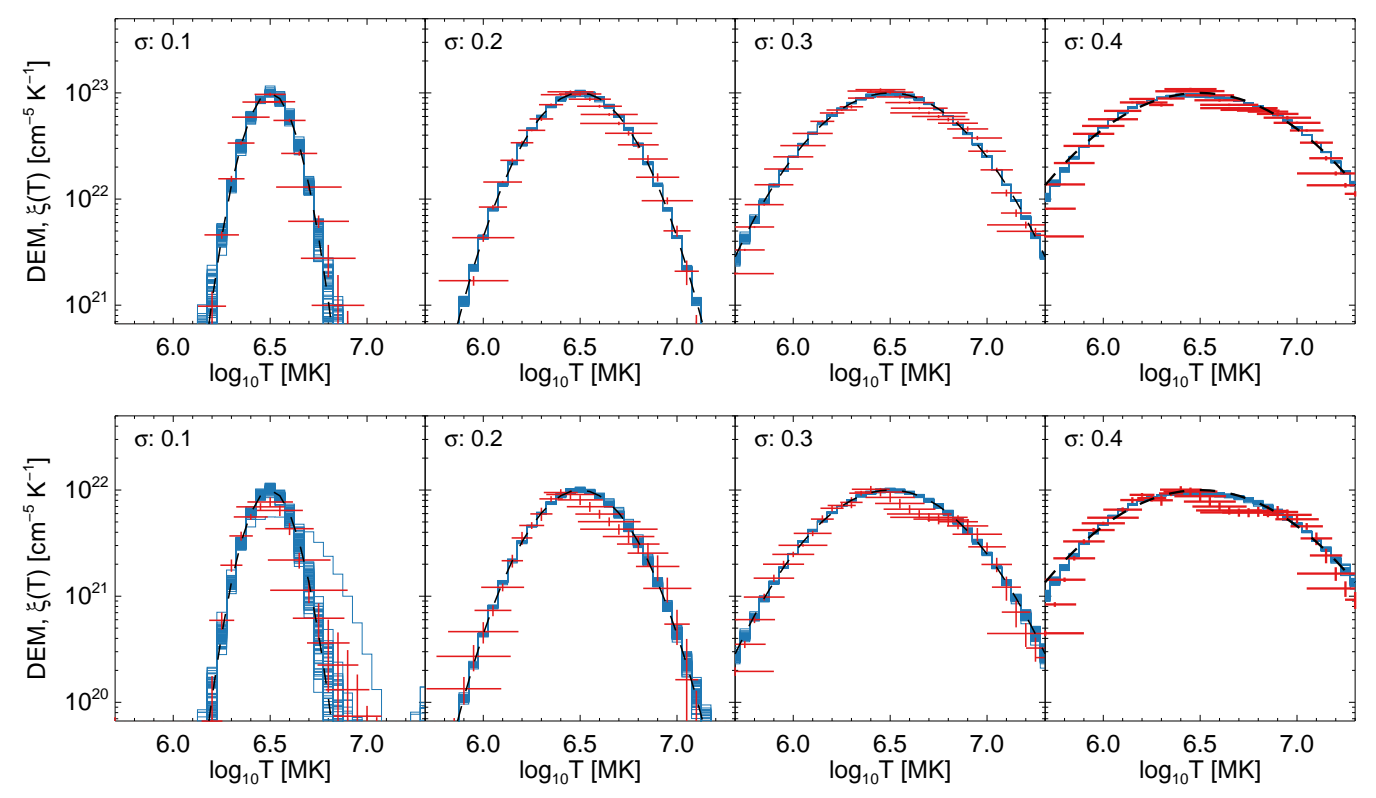

Fig. 8. DEMs recovered using regularized inversion (red error bars) and xrt_dem_i terative2 .pro (blue histograms, with 75 MC realisations) from simulated SDO/AIA data of Gaussian models (black dashed lines) of differing $\sigma$ (increasing left to right) and normalisation magnitude $\left(10^{23}\right.$ top row, $10^{22}$ bottom row).

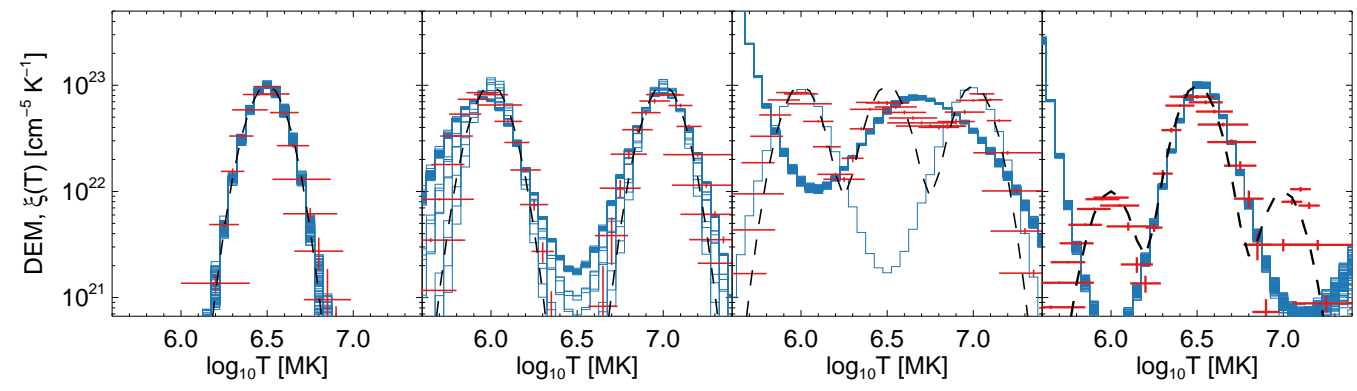

Fig. 9. DEMs recovered using regularized inversion (red error bars) and xrt_dem_iterative2 .pro (blue histograms, with 75 MC realisations) from simulated SDO/AIA data of models (black dashed lines) with a differing number of Gaussian components (left to right).

similar temperature ranges, for instance below $\log T \approx 5.9$ in the $\sigma_{T}=0.4$ model. We repeat the exercise but with model DEMs an order of magnitude smaller, $N_{0} /\left(\sqrt{2 \pi} \sigma_{T}\right)=10^{22} \mathrm{~cm}^{-5} \mathrm{~K}^{-1}$, shown in the bottom panels of Figure 8 . With the reduced signal to noise both methods produce poorer DEM solutions, particularly for the narrowest Gaussian model showing the expected degradation of the resolution recoverable with increasing noise. In this case one of the xrt_dem_iterative2 . pro solutions deviates greatly above $\log T \approx 6.7$. For the broader DEMs the iterative forward fitting method produces DEMs closer to the model than the regularized inversion, though the later is mostly consistent within the indicated error bars. For the regularized inversion a better or "smoother" solution could be archived by using a higher order constraint matrix however with real data there is no prior knowledge to the form of the emission and therefore no indication if such a constraint is actually better.

In Figure 9 we consider model DEMs constructed of several Gaussian components of width at the temperature resolution of SDO/AIA, $\sigma_{T} \approx 0.1$. The first panel shows the same single Gaussian shown in Figure 8 for comparison. With two Gaussian components with centroid temperatures of $\log T=6.0,7.0$ (second panel in Figure 9) the regularized solution closely matches the source model. The forward fitting approach (xrt_dem_i terative2 . pro) does get the general shape correct but produces a tall spread of solutions at the extremes of the temperature range chosen and in between the two Gaussian components. The former will be due to the reduced SDO/AIA response in this range but for the discrepancy about $\log T=6.5$ this is not the case. The situation is even worse for xrt_dem_iterative2.pro when three Gaussian components are considered (third panel in Figure 9, with centroid temperatures of $\log T=6.0,6.5,7.0)$ with the it producing a DEM solution completely different from the model. In one of the MC realisations the forward fitting method does recover two of the Gaussian components but still fails to recover the middle one. The regularized inversion method also has problems in recovering the model DEM but performs considerably better than xrt_dem_iterative2.pro, producing a DEM with three distinct components. Only between $\log T=6.4-6.8$ does it deviate from the model, failing to match the second Gaussian peak and subsequent minima. In the final panel of Figure 9 we again consider three Gaussian components but this time with those of centroid temperatures $\log T=6.0,7.0$ an order of magnitude smaller than the central component. This time xrt_dem_iterative2 . pro recovers the central component but 

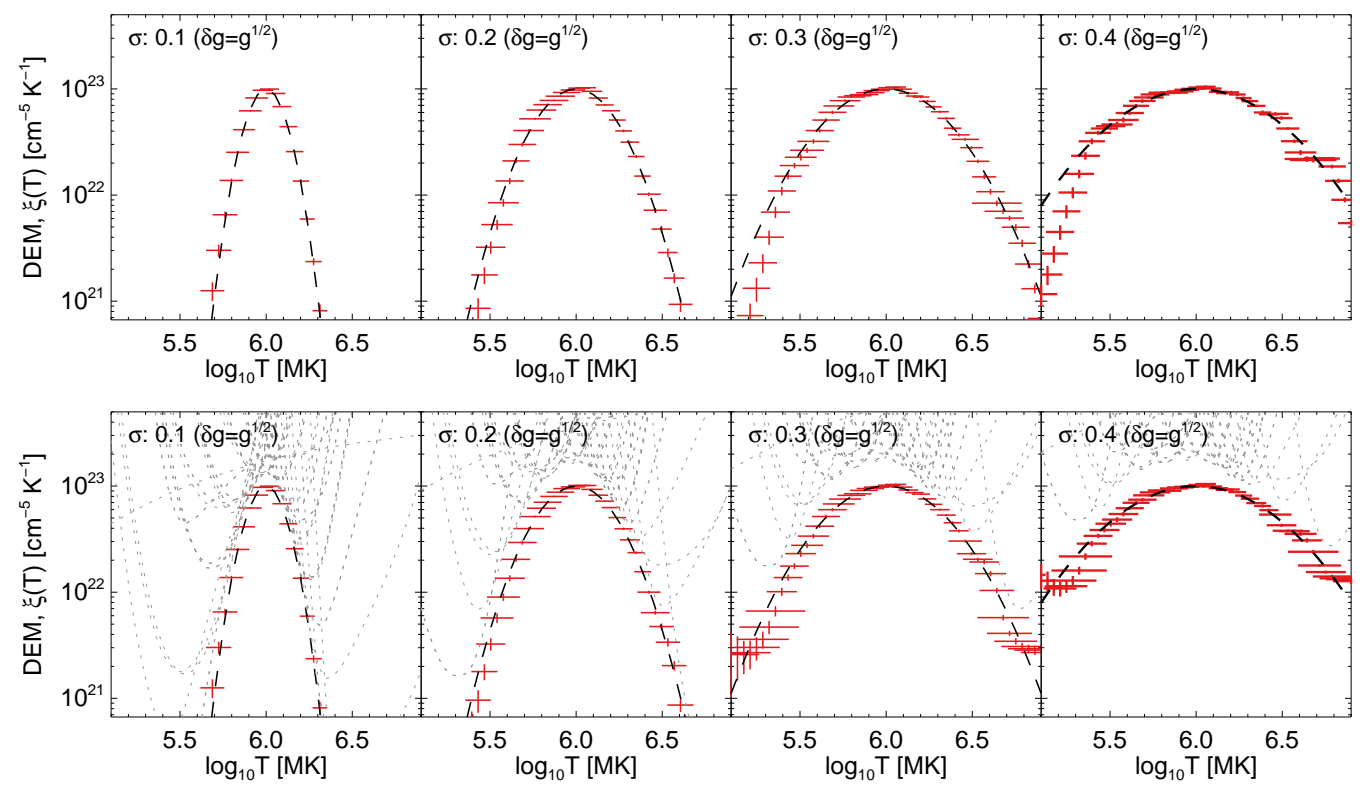

Fig. 10. DEMs recovered using regularized inversion (red error bars) from simulated Hinode/EIS data of Gaussian models (black dashed lines) of differing $\sigma$ (increasing left to right). Here the error in the line intensity is taken as the Poisson noise, which range between 0.2 to $11 \%$ of the line intensity. In the bottom row the minimum of the EM loci curves (grey dotted lines) has been used as the guess solution $\xi_{0}(T)$.
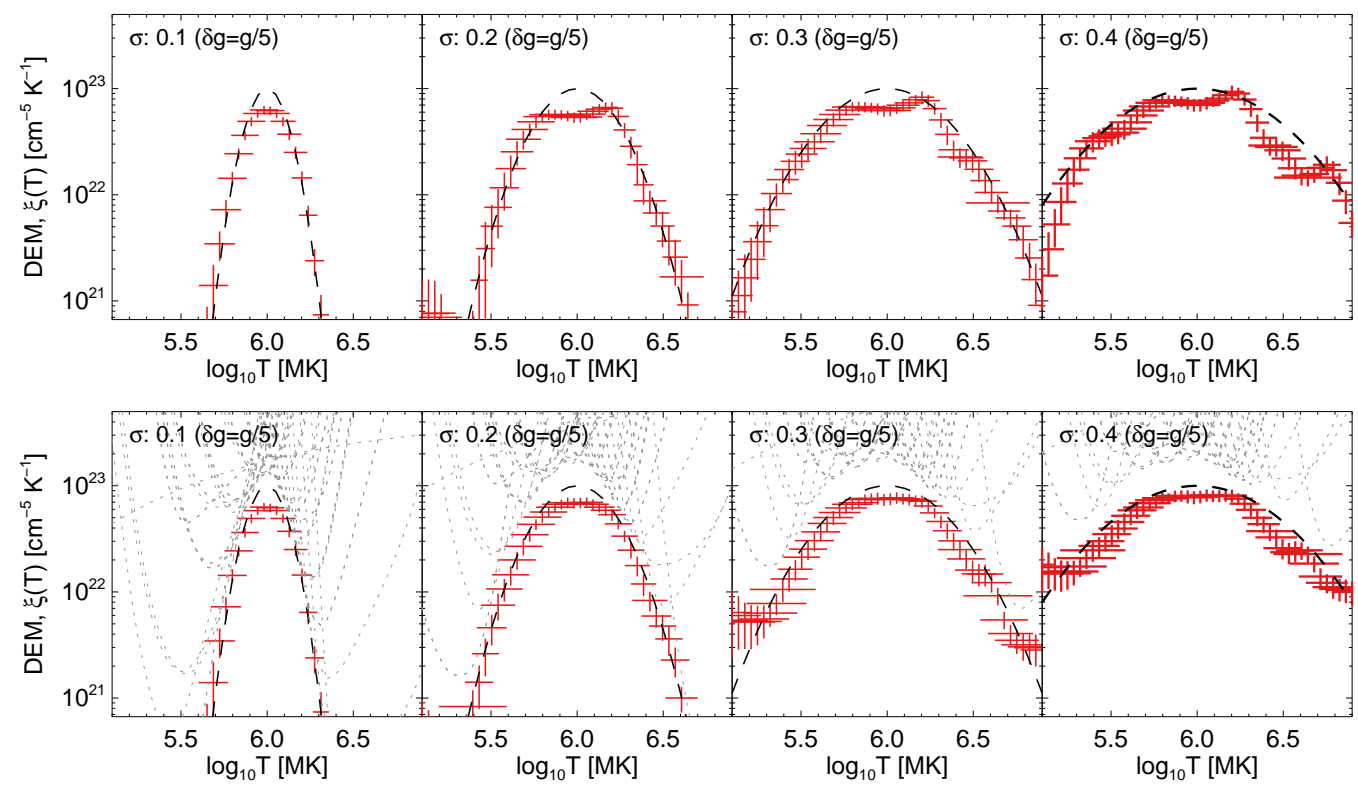

Fig. 11. DEMs recovered using regularized inversion (red error bars) from simulated Hinode/EIS data of Gaussian models (black dashed lines) of differing $\sigma$ (increasing left to right). Here the error is taken as $20 \%$ of the line intensity. In the bottom row the minimum of the EM loci curves (grey dotted lines) has been used as the guess solution $\xi_{0}(T)$.

completely missing the two smaller ones. Again the regularized inversion performs far better recovering three distinct components though slightly overestimates the centroid position of the hottest component.

\subsection{Hinode/EIS Simulated Data}

Spectroscopic observations, such as those from the EUV Imaging Spectrometer (EIS) on Hinode, can potentially recover the DEM better than broadband multi-filter observations given the significant number of temperature sensitive lines available.
The resulting observed line intensities and calculated contribution functions can be easily used with our regularization method as it only requires an observable, associated error and response as a function of temperature for a number of filters or lines. Here we use the atlas of EUV lines (observable with Hinode/EIS) from Landi \& Young (2009) with 48 of them shown in the bottom panel of Figure 1, These are lines emitted at cooler temperatures than observed by SDO/AIA and so we will consider Gaussian models with centroid temperatures between $\log T=$ 5.5 to 6.5 . 

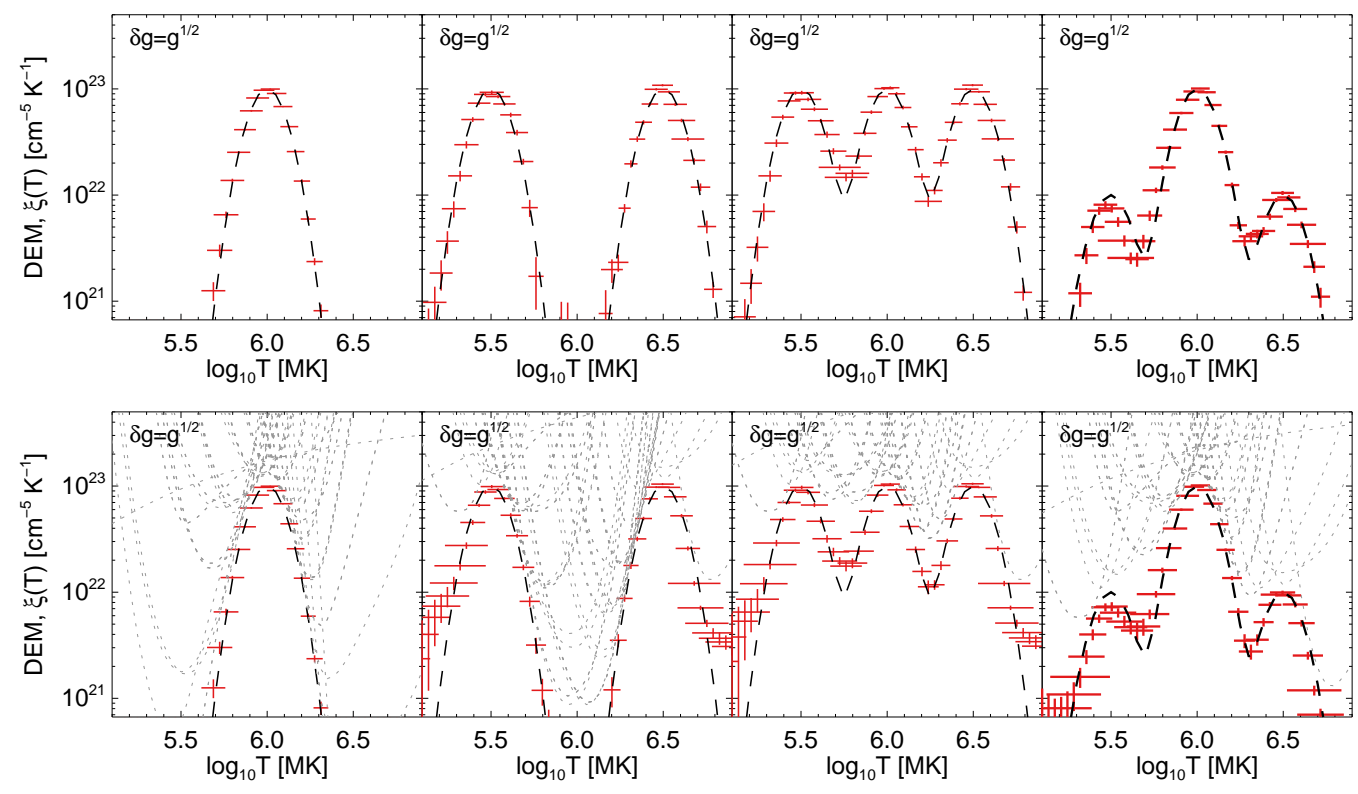

Fig. 12. DEMs recovered using regularized inversion (red error bars) from simulated Hinode/EIS data of multiple component Gaussian models (black dashed lines) of differing $\sigma$ (increasing left to right). Here the error in the line intensity is taken as the Poisson noise, which range between 0.2 to $11 \%$ of the line intensity. In the bottom row the minimum of the EM loci curves (grey dotted lines) has been used as the guess solution $\xi_{0}(T)$.
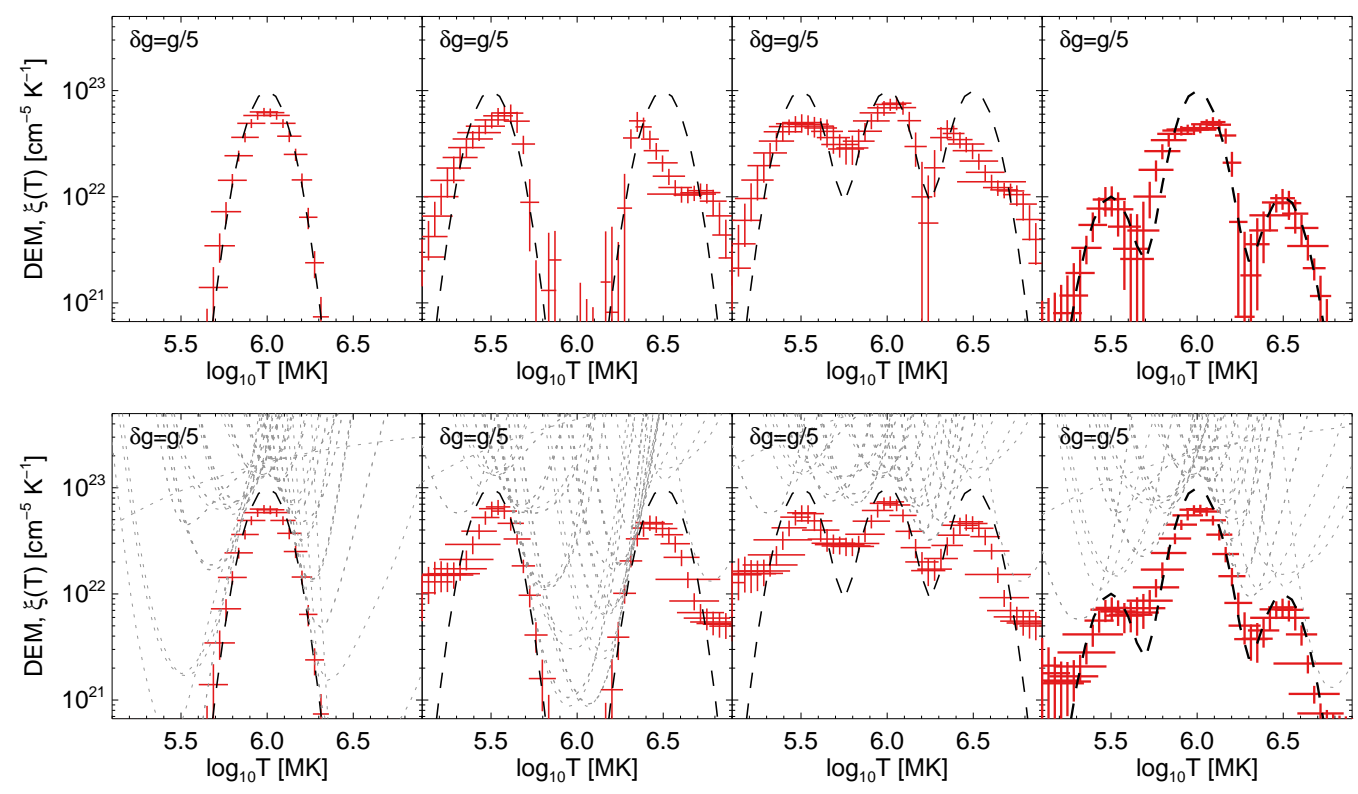

Fig. 13. DEMs recovered using regularized inversion (red error bars) from simulated Hinode/EIS data of multiple component Gaussian models (black dashed lines) of differing $\sigma$ (increasing left to right). Here the error is taken as $20 \%$ of the line intensity. In the bottom row the minimum of the EM loci curves (grey dotted lines) has been used as the guess solution $\xi_{0}(T)$.

The simulated line intensities are created as before with the pixel intensities for SDO/AIA but this time we consider not only the Poisson noise $\left(\delta g_{i}=\sqrt{g}\right)$ but also a systematic uncertainty that is a percentage of each line intensity. This corresponds to a temperature independent factor per line $c_{i}, \delta g_{i}=g_{i} / c_{i}$. We are using this approach as there are uncertainties in the relative (few percent), absolute (up to $22 \%$ ) and modelling $(\approx 10 \%)$ of the contribution functions for each line (private communication $P$. Young and Landi \& Young (2009)). Such a temperature independent systematic on the contribution function $\delta \mathbf{K}_{\mathbf{i}}=\mathbf{K}_{\mathbf{i}} / c_{i}$, where $c_{i}$ is the factor per line, is equivalent to the same system- atic on the observable $\delta g_{i}=g_{i} / c_{i}$, as discussed in $\$ 2.5$ So when calculating the error in the simulated observable for our chosen DEM we choose either the Poisson noise or a percentage error, in the latter case to represent this possibly dominant systematic uncertainty in the contribution functions.

In Figure 10 we show the regularized inversion of Gaussian model DEMs of different widths using a Poisson uncertainty on the line intensity, again using the zeroth-order constraint and $\alpha=1$ (i.e. $\chi^{2} \approx 1$ ). For the model DEMs used the uncertainties given by $\delta g_{i}=\sqrt{g}$ range from 0.2 and $11 \%$ of the line intensities. The regularized solution very closely matches the 

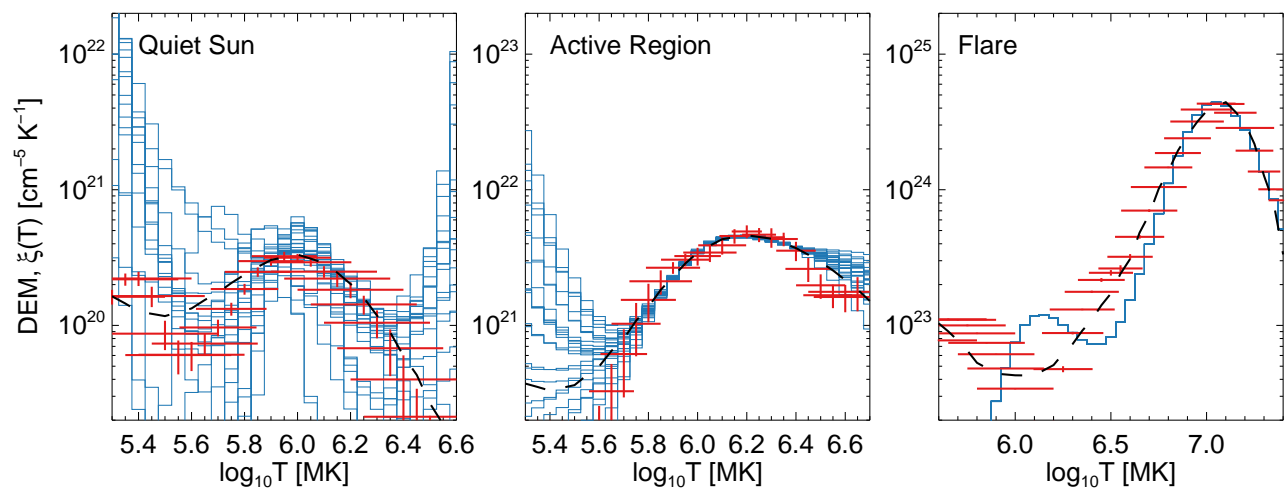

Fig. 14. Regularized DEMs (red error bars) and xrt_dem_i terative2 . pro solution (blue histograms, with $25 \mathrm{MC}$ realisations) for simulated SDO/AIA data of CHIANTI DEM models (dashed black lines, left to right: quiet sun, active region, M-Class flare).
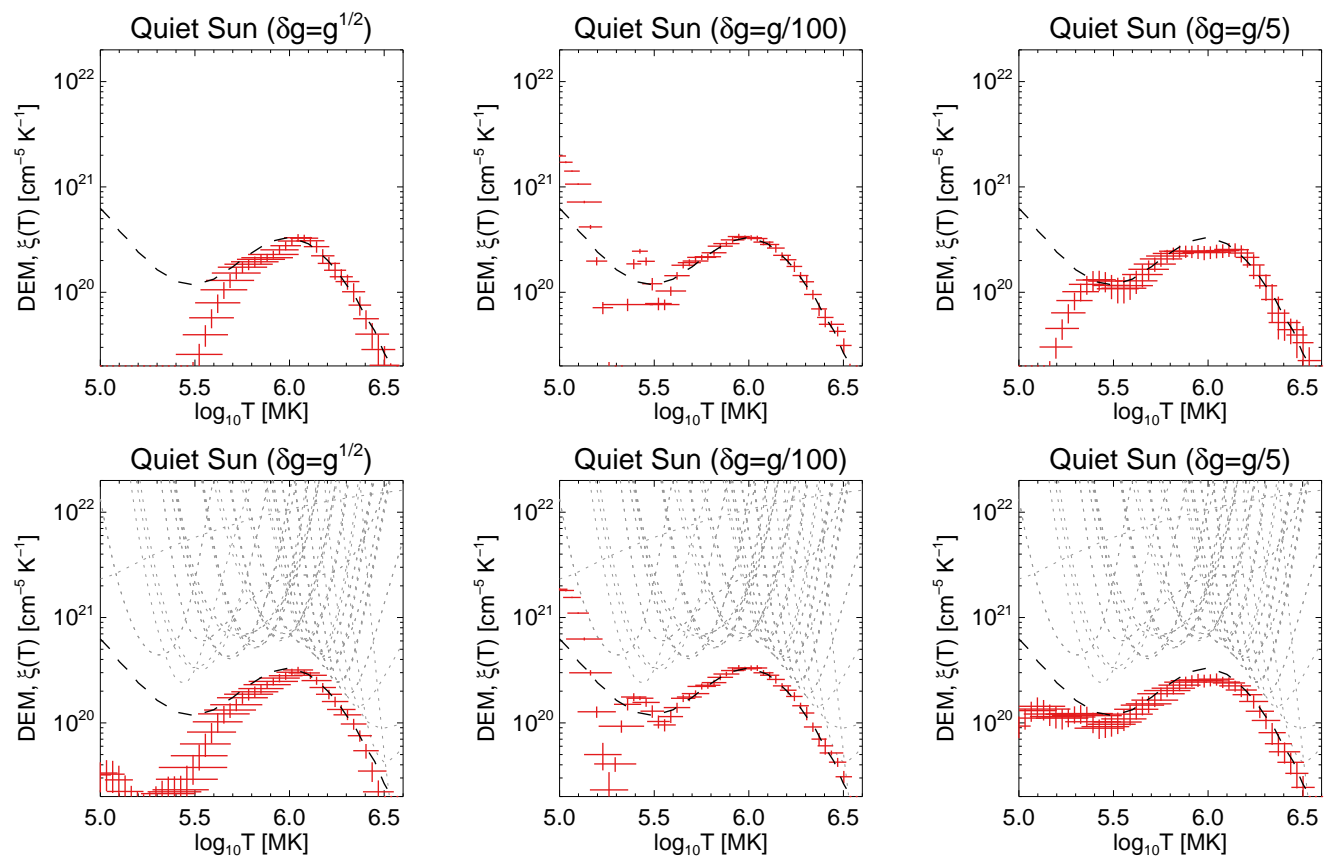

Fig. 15. Regularized DEMs (red error bars) for simulated Hinode/EIS lines of the CHIANTI quiet Sun DEM model. The error is taken as the Poisson noise and $1 \%$ and $20 \%$ of the line intensity (left to right). In the bottom row the minimum of the EM loci curves (grey dotted lines) was used as the guess solution $\xi_{0}(T)$.

model DEM for the narrowest cases (top left panels), matching the model down to the temperature resolution of the atomic data used to calculated the contribution functions, i.e. $\sigma_{T}=0.1$. For the wider Gaussian models (top right panels) the regularized solution recovers the model well about the peak emission but underestimates the emission at the lowest temperatures. These regularized DEMs were found using the two-stage approach of no initial guess solution $\xi_{0}(T)$ but we can also find the solutions using the minimum of the EM loci curves (grey dashed lines) as the guess solution (see $\$ 2.2$ ), shown in the bottom row of Figure 10. For the narrowest Gaussian models there is little difference in the regularized solutions. The only improvement here is in the time it takes to perform the computation since the regularization has only been calculated once. However even with the two-stage approach the DEM is computed in just a few seconds. For the wider Gaussian models the regularized DEM found using the guess solution recovers the model DEM better, particularly at lower temperatures.
This analysis is repeated but this time the error on the line intensities is taken to be $20 \%$ instead of the Poisson noise. With this increased uncertainty the regularization method does not recover the model DEMs as well, shown in Figure 11. For the narrowest DEM (top left panel) the regularized inversion recovers the majority of the model DEM but underestimates the peak emission and increases its width, the latter showing a reduction in temperature resolution due to noise. The use of the minimum of the EM loci curves as the guess solution $\xi_{0}(T)$ greatly improves the recovery of the source DEMs (bottom row) but still underestimates the peak emission in the narrowest cases. This demonstrates that the the temperature resolution had been inherently degraded by the increase in noise.

We now consider model DEMs constructed of several Gaussian components of width at the temperature resolution of the atomic data, $\sigma_{T} \approx 0.1$, shown with Poisson noise and then $20 \%$ uncertainty in the line intensity in Figures 12 and 13 . With the lower level of noise from the Poisson errors the regularized 

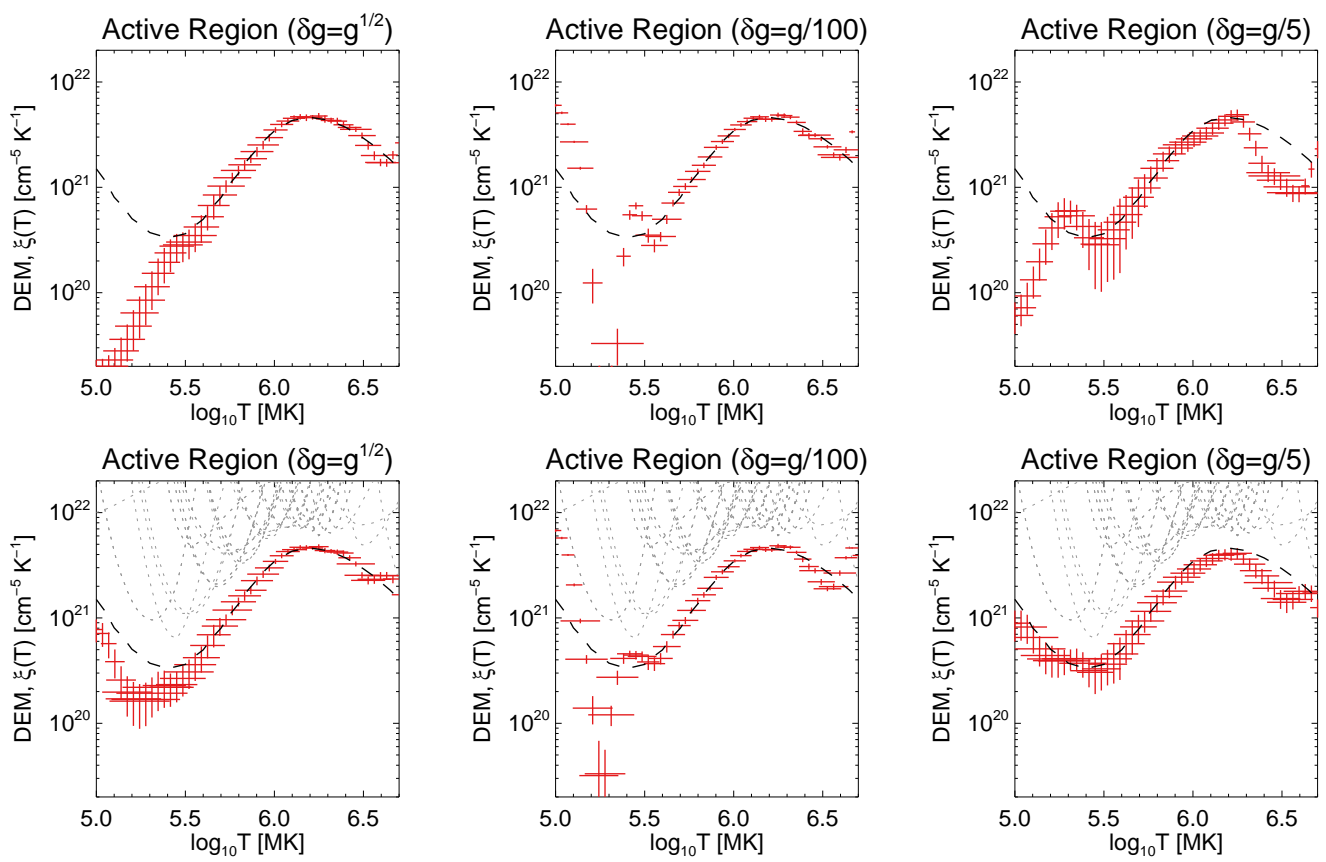

Fig. 16. Regularized DEMs (red error bars) for simulated Hinode/EIS lines of the CHIANTI active region DEM model. The error is taken as the Poisson noise and $1 \%$ and $20 \%$ of the line intensity (left to right). In the bottom row the minimum of the EM loci curves (grey dotted lines) has been used as the guess solution $\xi_{0}(T)$.

inversion recovers well the model DEMs (top row Figure 12), even in the cases with three Gaussian components. The use of the initial guess solution (bottom row Figure 12) generally does worse in recovering the DEMs at the extremes of the temperature ranges but produces similar results at the mid-range temperatures where the DEMs peak. With a larger error in the line intensity the regularized inversion struggles to recover the model DEMs (Figure 13). In all cases it is able to recover the correct number of distinct components in the DEMs but is unable to match the peak emission, often producing flatter solutions. The use of the initial guess solution does help recover the model DEMs better (bottom row Figure 13) but it still struggles with this noisy simulated data.

\section{Simulated Data: CHIANTI Model DEMs}

To test the regularization with more physically realistic DEMs we use those provided with the CHIANTI atomic database (Dere et al. 1997, 2009). We use the DEMs for the quiet Sun (del Zanna 1999), an active region (Andretta et al. 2003) and a M2-GOES Class flare (Dere \& Cook 1979). With SDO/AIA we test all three of these models (see $\$ 4.1$ ) but for the Hinode/EIS lines we only consider the quiet Sun and active region models (see $\$ 4.2$ ) since these lines are sensitive to temperatures predominantly below $\log T \approx 6.5$, lower than expected in a large flare. Again the regularized solutions were found using the zerothorder constraint and as $\alpha=1$ the resulting solutions have approximately $\chi^{2}=1$.

\subsection{SDO/AIA Simulated Data}

The regularized DEMs recovered from simulated SDO/AIA data of the CHIANTI model DEMs is shown in Figure 14. The first DEM shown is for the quiet Sun and the regularization recovers the DEM well but has very large error bars. This is understandable given the faint, and hence noisy, emission: the peak emis- sion for the quiet Sun DEM is about $10^{20} \mathrm{~cm}^{-5} \mathrm{~K}^{-1}$ which is over three orders of magnitude smaller that the Gaussian examples in \$3.1. In comparison, xrt_dem_iterative2 . pro poorly recovers the model DEM producing a large spread of the MC realisations. The active region model DEM (middle panel Figure 14) is better recovered than the quiet Sun model which is expected given that this DEM produces a stronger signal. Both methods have trouble in recovering the model DEM at the lowest temperatures $(\log T<5.7)$ where the emission is smallest: the regularization solution underestimating the emission, the iterative approach overestimating. For the flare DEM (right panel Figure 14) both methods recover the model well about the peak temperature $(\log T \approx 7.0)$ will small vertical uncertainties. This is due to the large emission from the flare, about 3 to 4 orders of magnitude larger than the quiet Sun and active region models. At lower temperatures the regularized solution matches the model DEM well though with a substantial horizontal spread. The xrt_dem_iterative2 .pro solutions deviate from the model below $\log T \approx 6.7$ and produces a false peak about $\log T \approx 6.1$ when in the model it is a minimum.

\subsection{Hinode/EIS Simulated Data}

The regularized DEM of the CHIANTI quiet Sun model using the Hinode/EIS lines is shown in Figure 15, where it has been calculated using a variety of errors in the line intensities and with/without the minimum of the EM loci as the initial guess solution. When no initial guess solution is used (top row) the regularization method is able to recover the main peak of emission (about $\log T \approx 6.0$ ) for the three different error cases, Poisson noise, $1 \%$ and $20 \%$. Only with the $1 \%$ errors on the line intensities does the regularized solution properly recover that the emission increases with decreasing temperature but does not match it very well (middle panel). Although it should be noted that this model produces very weak emission and the DEM recoverable from these multiple Hinode/EIS lines is considerably better than 

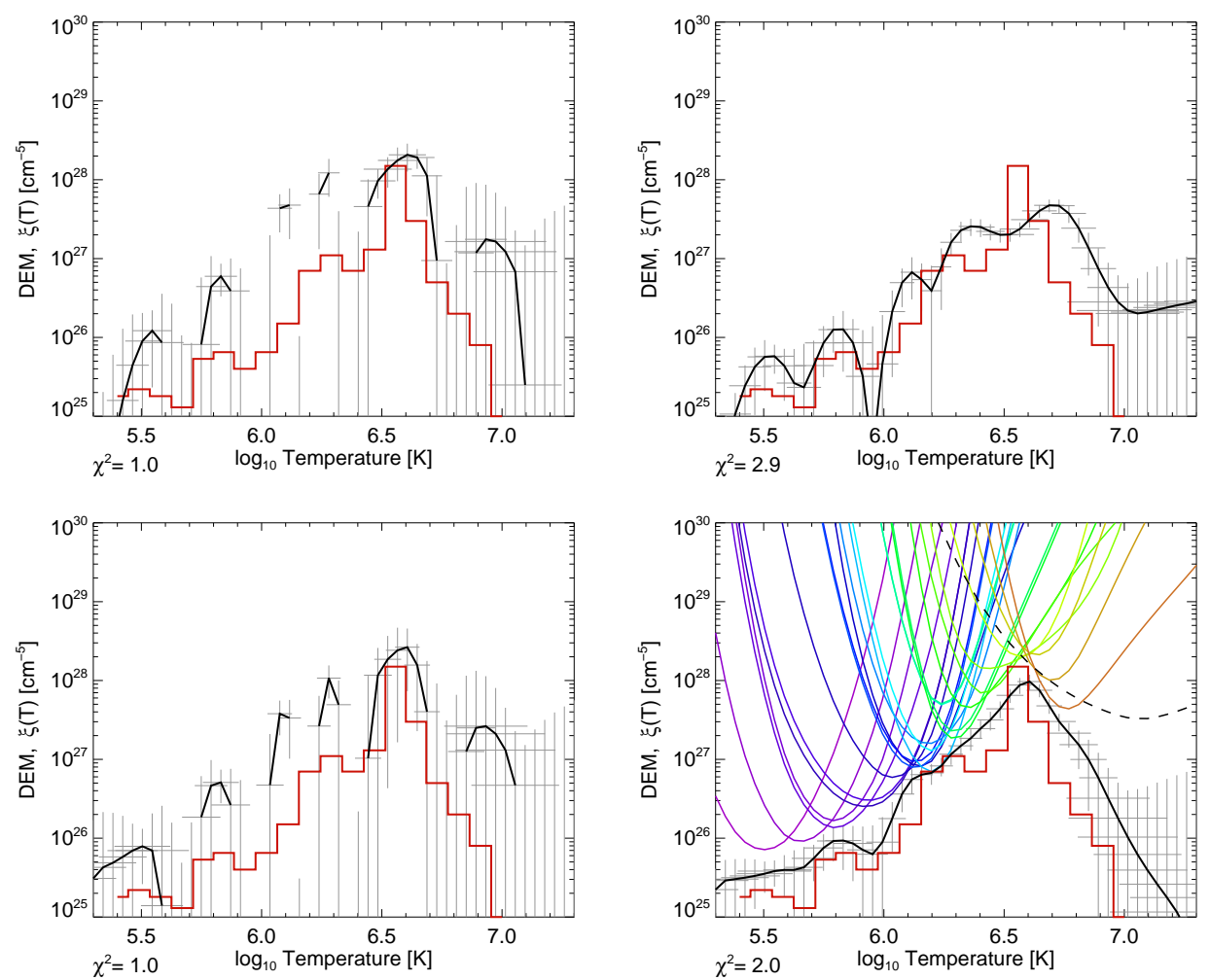

Fig. 17. Regularized emission measure distributions (black solid line and grey error bars) for the active region core observations made by Warren et al. (2010), their MCMC solution (red histogram) is shown for comparison. In the right-hand panels the positivity constraint was used and hence larger $\chi^{2}$ values are achieved by the regularized solutions. The EM loci curves for the Hinode/EIS (coloured lines) and Hinode/XRT filter (black dashed line) are shown in the bottom left panel and their minimum was used as an initial guess solution for the regularizations shown in the bottom row.

with the SDO/AIA simulated data (left panel Figure 14). When the minimum of the EM loci curves is used as the initial guess solution (bottom row, Figure 15) the regularized solution does recover more of the model DEM but the problems with the low temperature component persist.

In Figure 16 we show the same analysis but this time for the CHIANTI active region DEM model. The $1 \%$ error case again well recovers the majority of model DEM but struggles at low and high temperatures when higher, and more realistic, errors in the line intensities are used. When the minimum of the EM loci curves are used as the initial guess solution (bottom row, Figure 16 the regularization method does considerably better by impressively recovering the model DEM even in the situations with larger errors.

\section{Real Data: Hinode/XRT and EIS}

To test the performance of the regularization on real data we use the observations of an active region core with Hinode/EIS and XRT from Warren et al. (2010). This article provides the line intensities, with errors (which were $20 \%$ to $23 \%$ ), for 24 EIS lines and one XRT filter (Al-thick) (see Table 1, Warren et al. (2010)) and computed the DEM (Figure 4, red histogram, Warren et al. (2010)) using the MCMC method packaged with PINTofALE (Kashyap \& Drake 1998). Using the information given we use CHIANTI to calculate the contribution functions for the 24 EIS lines, and with the Hinode/XRT temperature response function for the Al-thick filter (Golub et al. 2007), and the quoted intensities and errors we calculate the zeroth-order regularized solu- tions and $\alpha=1$ (shown in Figure 17). Here we have the regularized solutions found both with and without the positivity constraint (left vs right columns) and the initial guess solution from the minimum of the EM loci curves (top vs bottom rows). Shown for comparison is the MCMC solution found by Warren et al. (2010). Note that for all the regularized solutions the resulting $\mathbf{R}_{\lambda} \mathbf{K}$ matrix was almost diagonal over $\log T \approx 5.5-6.9$ indicating that the regularization has successfully worked over this temperature range. With no positivity constraint (left-hand panels) the regularized solution is highly oscillatory between positive and negative values, an indication that this is an over-regularized solution (Craig 1977; Bertero et al. 1985). Although, the regularization does produce a maximum at the same temperature as the MCMC method. The use of the initial guess solution (bottom left panel Figure 17) results in minimal changes to the recovered DEM. The positivity constraint produces a closer match to the MCMC solution. When no initial guess solution is used (top right panel) a highly oscillatory DEM is recovered and deviates from the MCMC solution at the peak temperature and above. When the minimum of the EM loci curves are used as the initial guess solution (bottom right panel) a smaller $\chi^{2}$ is achieved while still having a positive solution. This regularized DEM is a close match to MCMC solution, even more so when one includes the spread of the DEM found from $250 \mathrm{MC}$ solutions (shown in Figure 4, Warren et al. (2010)). The crucial difference though is in the computation time: the regularized solution is found within a few seconds, the MCMC method taking orders of magnitude longer. 


\section{Discussion \& Conclusions}

In this work, we have applied a regularized inversion technique developed for RHESSI HXR analysis to multi-filter observations of hot solar plasma with Hinode/EIS, XRT and SDO/AIA. This method successfully recovers a variety of model DEMs from different simulated broadband and spectroscopic data of varying noise and uncertainty. It is implemented $\sqrt[3]{\text { using General Singular }}$ Value Decomposition (GSVD) and this has several advantages over previous approaches used to find the regularized inversion of solar data (i.e Craig 1977; Judge et al. 1997):

1. It reliably recovers the DEM even from noisy data and using only the weakest (yet mostly physically justifiable) constraint, zeroth-order $L_{0}$;

2. This method naturally determines the confidence interval for the regularized solution by calculating both the vertical and horizontal error bars allowing an objective assessment of the quality of the data, temperature response functions and DEM. The use of the $\mathbf{R}_{\lambda} \mathbf{K}$ matrix also provides additional information about the robustness of the regularized solution;

3. It is computationally very quick, with DEMs recovered from SDO/AIA data in about a second or less and a few seconds when a large number of spectral lines are used, such as with Hinode/EIS. This is crucial considering the colossal amount of ever increasing data that is being accumulated by SDO, Hinode and expected from future missions.

4. The recovery of a positive solution can be guaranteed though this approach can hide issues with the data and response functions.

Misconceptions about the problems with inversion techniques (such as the smoothness criteria) still exist (e.g. Landi et al. 2011) but the examples shown in this paper clearly demonstrate that these views are misplaced and our regularized inversion approach can robustly recover a variety of DEMs. Moreover the ability to easily determine the temperature uncertainty is a major improvement over other methods, crucial when trying to test the possibly isothermal nature of solar plasma.

DEMs are a very useful tool for characterising the temperature distribution of the corona and trying to reveal the properties of the mechanisms that are heating the plasma. However there are several caveats to the reconstructed DEMs which mean they should not be over-interpreted. Any method that recovers a DEM from a set of real data cannot guarantee that it is the actual solution given the possible uncertainties/errors in the data and temperature response functions (either instrumental or in the atomic physics). The use of more than one method is highly recommended as this would highlight any issues and artifacts of a particular approach and demonstrate the role of the uncertainty in the data on the solution. The computational speed of our regularization method makes it a minor burden to include when using other techniques and also allows fast exploration of the effect of the uncertainties on the DEM, as demonstrated for the simulated data in this paper. Even if several techniques produce the same DEM, with similar uncertainties, this still leaves the possibility of there being errors in the temperature response functions due to mis-calibration of the instrument or information in the atomic data being absent or erroneous. There is a continuous effort to improve the atomic physics data, through the sterling work of the CHIANTI team and others. At the moment there are known issues with the SDO/AIA response functions (e.g.

3 The code written in IDL requiring SSW is available online: http://www.astro.gla.ac.uk/ iain/demreg/
Aschwanden \& Boerner 2011) and the effect of this can be tested by simulating SDO/AIA data using the "correct" response but recover the DEM from this data using the erroneous responses. However if the emission is not optically thin or not in thermal equilibrium the DEM and response functions are not appropriate to describe the temperature distribution of the plasma.

All these problems are compounded by the vast quantities of solar data now available resulting in the current need for a variety of tools to investigate DEMs quickly, transparently and easily. The use of simulated data is a vital approach to investigate the role of each of these issues and combined with a computationally fast algorithm that provides error estimates, such as our regularization method, should provide a quicker determination of the reliability of DEMs and what can be interpreted from them.

Acknowledgements. This work is supported by a STFC grant (IGH, EPK). Financial support by the European Commission through the FP7 HESPE Network is gratefully acknowledged. We would like to thank the referee for their constructive criticisms that helped us greatly improve this paper.

\section{References}

Andretta, V., Del Zanna, G., \& Jordan, S. D. 2003, A\&A, 400, 737 Aschwanden, M. J. \& Boerner, P. 2011, ApJ, 732, 81

Bertero, M., DeMol, C., \& Pike, E. R. 1985, Inverse Problems, 1, 301

Bertero, M., DeMol, C., \& Pike, E. R. 1988, Inverse Problems, 4, 573

Bertero, M. \& Dovi, V. 1981, Optica Acta, 28, 1635

Boerner, P., Edwards, C., Lemen, J., et al. 2011, Sol. Phys., 193

Brosius, J. W., Davila, J. M., Thomas, R. J., \& Monsignori-Fossi, B. C. 1996, ApJS, 106, 143

Brown, J. C., Emslie, A. G., Holman, G. D., et al. 2006, ApJ, 643, 523

Craig, I. J. D. 1977, A\&A, 61, 575

Craig, I. J. D. \& Brown, J. C. 1986, Inverse problems in astronomy (Bristol: Hilger)

Culhane, J. L., Harra, L. K., James, A. M., et al. 2007, Sol. Phys., 243, 19

de Villiers, G. D., McNally, B., \& Pike, E. R. 1999, Inverse Problems, 15, 615 del Zanna, G. 1999, PhD thesis, , Univ. of Central Lancashire, (1999)

Dere, K. P. \& Cook, J. W. 1979, ApJ, 229, 772

Dere, K. P., Landi, E., Mason, H. E., Monsignori Fossi, B. C., \& Young, P. R. 1997, A\&AS, 125, 149

Dere, K. P., Landi, E., Young, P. R., et al. 2009, A\&A, 498, 915

Fletcher, L., Dennis, B. R., Hudson, H. S., et al. 2011, Space Sci. Rev., 159, 19

Golub, L., Deluca, E., Austin, G., et al. 2007, Sol. Phys., 243, 63

Golub, L., Deluca, E. E., Sette, A., \& Weber, M. 2004, in Astronomical Society of the Pacific Conference Series, Vol. 325, The Solar-B Mission and the Forefront of Solar Physics, ed. T. Sakurai \& T. Sekii, 217-+

Goryaev, F. F., Parenti, S., Urnov, A. M., et al. 2010, A\&A, 523, A44+

Hannah, I. G., Hudson, H. S., Battaglia, M., et al. 2011, Space Sci. Rev., 159, 263

Hansen, P. C. 1992, Inverse Problems, 8, 849

Hirayama, T. 1974, Sol. Phys., 34, 323

Judge, P. G. 2010, ApJ, 708, 1238

Judge, P. G., Hubeny, V., \& Brown, J. C. 1997, ApJ, 475, 275

Kashyap, V. \& Drake, J. J. 1998, ApJ, 503, 450

Kontar, E. P., Piana, M., Massone, A. M., Emslie, A. G., \& Brown, J. C. 2004, Sol. Phys., 225, 293

Landi, E., Reale, F., \& Testa, P. 2011, ArXiv e-prints

Landi, E. \& Young, P. R. 2009, ApJ, 706, 1

Lemen, J. R., Title, A. M., Akin, D. J., et al. 2011, Sol. Phys., 172

Lin, R. P., Dennis, B. R., Hurford, G. J., et al. 2002, Sol. Phys., 210, 3

Mariska, J. T. 1992, The solar transition region, ed. Mariska, J. T.

Massone, A. M., Emslie, A. G., Kontar, E. P., et al. 2004, ApJ, 613, 1233

McIntosh, S. W., Charbonneau, P., \& Brown, J. C. 2000, ApJ, 529, 1115

Monsignori Fossi, B. C. \& Landini, M. 1991, Advances in Space Research, 11, 281

Monsignori-Fossi, B. C. \& Landini, M. 1992, Mem. Soc. Astron. Italiana, 63, 767

Morozov, V. 1967, Doklady Akademii Nauk SSSR, 175, 1225

O'Dwyer, B., Del Zanna, G., Mason, H. E., et al. 2011, A\&A, 525, A137+

Parenti, S., Bromage, B. J. I., Poletto, G., et al. 2000, A\&A, 363, 800

Parker, E. N. 1988, ApJ, 330, 474 
Phillips, K. J. H., Feldman, U., \& Landi, E. 2008, Ultraviolet and X-ray Spectroscopy of the Solar Atmosphere, ed. Phillips, K. J. H., Feldman, U., $\&$ Landi, E. (Cambridge University Press)

Piana, M. \& Bertero, M. 1997, Inverse Problems, 13, 441

Piana, M., Massone, A. M., Kontar, E. P., et al. 2003, ApJ, 595, L127

Prato, M., Piana, M., Brown, J. C., et al. 2006, Sol. Phys., 237, 61

Press, W. H., Teukolsky, S. A., Vetterling, W. T., \& Flannery, B. P. 1992 , Numerical recipes in C. The art of scientific computing, ed. Press, W. H., Teukolsky, S. A., Vetterling, W. T., \& Flannery, B. P.

Reale, F., Testa, P., Klimchuk, J. A., \& Parenti, S. 2009, ApJ, 698, 756

Schmelz, J. T., Jenkins, B. S., Worley, B. T., et al. 2011, ApJ, 731, 49

Schmitt, J. H. M. M., Drake, J. J., Stern, R. A., \& Haisch, B. M. 1996, ApJ, 457, 882

Siarkowski, M., Falewicz, R., Kepa, A., \& Rudawy, P. 2008, Annales Geophysicae, 26, 2999

Tikhonov, A. N. 1963, Soviet Math. Dokl., 4, 1035

Warren, H. P., Winebarger, A. R., \& Brooks, D. H. 2010, ApJ, 711, 228

Weber, M. 2009, in Astronomical Society of the Pacific Conference Series, Vol. 415, The Second Hinode Science Meeting: Beyond Discovery-Toward Understanding, ed. B. Lites, M. Cheung, T. Magara, J. Mariska, \& K. Reeves, $32-+$

Weber, M. A., Deluca, E. E., Golub, L., \& Sette, A. L. 2004, in IAU Symposium, Vol. 223, Multi-Wavelength Investigations of Solar Activity, ed. A. V. Stepanov, E. E. Benevolenskaya, \& A. G. Kosovichev, 321-328

Weber, M. A., Schmelz, J. T., DeLuca, E. E., \& Roames, J. K. 2005, ApJ, 635, L101

Woods, T. N., Eparvier, F. G., Hock, R., et al. 2010, Sol. Phys., 3 